\title{
Location and function of Plasmodium kinesins: key roles in parasite proliferation, polarity, and transmission
}

Mohammad Zeeshan ${ }^{1 *}$, Ravish Rashpa ${ }^{2 \dagger}$, David J Ferguson ${ }^{3,4 \dagger}$, Steven Abel ${ }^{5 \dagger}$, Zeinab Chahine $^{5}$, Declan Brady ${ }^{1}$, Sue Vaughan ${ }^{3}$, Carolyn A. Moores ${ }^{6}$, Karine G. Le Roch ${ }^{5}$, Mathieu Brochet ${ }^{2}$, Anthony A. Holder ${ }^{7}$ and Rita Tewari ${ }^{1 *}$

${ }^{1}$ University of Nottingham, School of Life Sciences, Nottingham, United Kingdom.

${ }^{2}$ University of Geneva, Faculty of Medicine, Geneva, Switzerland.

${ }^{3}$ Oxford Brookes University, Department of Biological and Medical Sciences, Oxford, United Kingdom.

${ }^{4}$ University of Oxford, John Radcliffe Hospital, Nuffield Department of Clinical Laboratory Science, Oxford, United Kingdom.

${ }^{5}$ Department of Molecular, Cell and Systems Biology, University of California Riverside, 900 University Ave., Riverside, USA.

${ }^{6}$ Institute of Structural and Molecular Biology, Department of Biological Sciences, Birkbeck College, London, United Kingdom.

${ }^{7}$ The Francis Crick Institute, Malaria Parasitology Laboratory, London, United Kingdom.

${ }^{\dagger}$ Contributed equally

${ }^{*}$ For correspondence

Rita Tewari: rita.tewari@nottingham.ac.uk

Mohammad Zeeshan: zeeshanmf@gmail.com

Short title: Genome-wide analysis of Plasmodium kinesins 
Abstract

Kinesins are microtubule-based motors important in cell division, motility, polarity and intracellular transport in many eukaryotes, but poorly studied in eukaryotic pathogens. Plasmodium spp., the causative agents of malaria, are divergent eukaryotes with atypical aspects of cell division and plasticity of morphology throughout the lifecycle in both mammalian and mosquito hosts. Here we describe a genome-wide screen of Plasmodium kinesins, revealing diverse subcellular locations and functions in spindle assembly, axoneme formation and cell morphology. Surprisingly, only kinesin-13 has an essential role for growth in the mammalian host while the other eight kinesins are required during the proliferative and invasive stages of parasite transmission through the mosquito vector. In-depth analyses of kinesin-13 and kinesin-20 revealed functions in microtubule dynamics during apical polarity formation, spindle assembly and axoneme biogenesis. This comprehensive study will inform the targeting of microtubule motors for therapeutic intervention in malaria. 


\section{Introduction}

Kinesins are microtubule (MT)-based motor proteins that use energy from the hydrolysis of ATP and function in various cellular processes including intracellular transport, mitotic spindle formation and chromosome segregation during cell division, and the organisation of cell polarity and cytoskeletal features associated with motility (Konjikusic et al., 2021; Verhey and Hammond, 2009). There are 14 to16 kinesin subfamilies categorised in eukaryotes according to the primary sequences of the motor domain, with similar biological roles also established by in vitro studies, and in vivo phenotypes for subfamily members (Hirokawa and Tanaka, 2015; Konjikusic et al., 2021; Yount et al., 2015). Kinesin subfamilies that regulate MT dynamics, such as kinesin- 8 and -13 , are found in most eukaryotes including primitive and evolutionarily divergent eukaryotes (Vicente and Wordeman, 2015; Wickstead et al., 2010). Although there is an extensive literature with various bioinformatic and molecular investigations of kinesins, information on these molecular motors is sparse in deep rooted pathogenic eukaryotes including Plasmodium spp. and other Apicomplexa, Giardia spp, and trypanosomes (Vicente and Wordeman, 2015). These primitive eukaryotes have a flagellate stage in their life cycle and may have a complex MT-associated cytoskeleton (Wickstead and Gull, 2011) indicating the importance of MT-based motor proteins in their development.

Plasmodium spp., the causative agents of malaria belong to the phylum Apicomplexa. They are ancient haploid unicellular eukaryotes with a number of morphologically diverse proliferative stages during the complex life cycle in various cells, tissues and organs of their vertebrate and invertebrate hosts (Fig. 1A) (Sinden, 1991; Zeeshan et al., 2020b). In the mammalian host the parasite proliferates within liver and red blood cells by repeated cycles of closed endomitotic division while retaining an intact nuclear membrane, with cytokinesis following the final nuclear division, in a process termed schizogony, to produce multiple infective haploid merozoites (Fig. 1A). In the cyclic, asexual proliferative blood stage, merozoites invade red blood cells (RBCs), developing through ring and trophozoite stages into schizonts (Sinden, 1991; Zeeshan et al., 2020b). DNA replication and asynchronous nuclear division form a polyploid cell followed by a final synchronised round of S-phase, karyokinesis and subsequent cytokinesis (Gubbels et al., 2020). Some of these haploid parasites in the RBC arrest and commit to sexual development as gametocytes (Fig. 1A). Gametocytes develop no further unless ingested in a blood meal by a female mosquito (the invertebrate host). Environmental conditions in the mosquito gut (lower temperature, higher alkalinity and the presence of xanthurenic acid) activate gametogenesis (Billker et al., 1998; Sinden et al., 1978). Female gametocytes produce a single, round, extracellular haploid female gamete 15 min after activation, without many apparent physiological or morphological changes (Sinden et al., 1978). Male gametogenesis is also very rapid and complete within 12 to $15 \mathrm{~min}$ after activation, but much more profound, with two major parallel events (Sinden et al., 1978). In three rounds of closed mitosis, DNA replication and chromosome segregation produce an $8 \mathrm{~N}$ genome, which is followed by nuclear division and cytokinesis. In the cytoplasm axoneme assembly and maturation occur, leading to the formation of flagellate haploid male gametes in a process termed exflagellation (Sinden, 1991; Zeeshan et al., 2020b). The motile male gamete finds and fertilises the female gamete, and the resultant zygote differentiates through six distinct stages (I to VI) into a banana-shaped, invasive motile ookinete with a distinct apical polarity and conoidassociated proteins (Janse et al., 1985; Koreny et al., 2021; Zeeshan et al., 2020b). At the same time, in the first stage of meiosis, the DNA is duplicated and the now tetraploid ookinete develops over a $24 \mathrm{~h}$ period in the mosquito gut (Janse et al., 1985; Zeeshan et 
al., 2020b), before traversing the mosquito gut wall and forming an oocyst under the basal lamina. Within the oocyst, sporogony, which is a form of endomitosis, produces many haploid sporozoites (Schrevel et al., 1977; Sinden and Strong, 1978). Sporozoites are motile and invasive polarised cells that migrate to and invade the salivary glands, so that the bite of the infected mosquito injects them into the next mammalian host (Graumans et al., 2020). Overall, the complete life cycle of the malaria parasite is characterised by varied morphological differences in size and shape, together with various modes of cell division and proliferation (Fig. 1A).

In a recent bioinformatic analysis of kinesins in Apicomplexa, we found nine kinesins encoded in the Plasmodium berghei genome, with members of three conserved kinesin subfamilies (kinesin-5, -8B, -8X and -13); kinesin-4, -15 and -20; and two Apicomplexa-enriched kinesins: kinesin-X3 and -X4 (Zeeshan et al., 2019b). Surprisingly, kinesin-5, $-8 \mathrm{X}$ and $-8 \mathrm{~B}$ were not essential for blood stage proliferation (Zeeshan et al., 2020a; Zeeshan et al., 2019a; Zeeshan et al., 2019b). However, deletion of kinesin-5, which codes for a protein clearly co-located with the spindle apparatus in all proliferative stages, affected the production of infective sporozoites (Zeeshan et al., 2020a). Kinesin-8X was required for endomitotic proliferation in oocysts, and kinesin-8B deletion resulted in a defect in axoneme biogenesis during male gametogenesis (Depoix et al., 2020; Zeeshan et al., 2019a; Zeeshan et al., 2019b).

Here, we present a genome-wide screen of all $P$. berghei kinesins, including additional analyses of previously studied kinesin-5, -8B and $-8 \mathrm{X}$ (Zeeshan et al., 2020a; Zeeshan et al., 2019a; Zeeshan et al., 2019b), using gene-targeting approaches, live-cell imaging, ultrastructure expansion microscopy and electron microscopy, and RNA-seq and ChIP-seq analyses. We examine the subcellular location of each kinesin using a protein endogenously tagged at the C-terminus with GFP, revealing a differential localisation of kinesins in mitotic and meiotic stages and a pellicular and polar location in certain invasive stages. Eight of the nine kinesin genes are required only for parasite transmission through the mosquito vector, during the sexual and sporogony stages. Only kinesin-13 is likely essential during blood stage schizogony. Kinesin-X3 and $-\mathrm{X} 4$, which are largely Apicomplexa-specific, show an interesting location on ookinete and sporozoite surface pellicle (-X3) and axonemes during male gametogenesis (-X4), respectively. These kinesins seem to have evolved in Apicomplexa for different MTbased structures such as the axoneme of flagella and a cytoskeletal scaffold to establish and maintain cell polarity, shape, and motility. An in-depth analysis of kinesin-13 and -20 revealed distinct subcellular locations and functions in MT spindle assembly and formation, axoneme assembly and cell polarity. Kinesin- 20 was associated with a striking ring-like structure during zygote to ookinete differentiation and deletion of the kinesin-20 gene revealed a function in the morphology and motility of the ookinete. Ultrastructure expansion methods and electron microscopic analysis showed a defect arising from the extent and organization of subpellicular MTs. Kinesin-13 is expressed at all proliferative stages of the life cycle, and it associates with the kinetochore. A kinesin-13 genetic knockdown affected MT dynamics during spindle formation and axoneme assembly in male gametocytes, and subpellicular MT organization in ookinetes.

This comprehensive study of all $P$. berghei kinesins showed that most are required during parasite development within the mosquito vector where there are several morphological forms of the parasitecell, with differentiation between invasive and proliferative stages. Kinesin-13 is the only kinesin essential for both asexual blood stages and sexual stages. These findings will inform a strategy to target MT motors for therapeutic intervention against malaria. 


\section{Results}

Live-cell imaging of all Plasmodium kinesins reveals diverse locations during cell division, differentiation, and pellicle formation throughout the life cycle.

To investigate the expression and subcellular location of kinesins throughout the $P$. berghei life cycle, we generated transgenic parasite lines by single crossover recombination at the 3' end of each gene to express a fusion protein with a C-terminal GFP-tag (fig. S1A). PCR analysis of genomic DNA from each line, using locus-specific diagnostic primers, indicated correct integration of the GFP sequence (fig. S1B). Each kinesin-GFP parasite line completed the full life cycle with no detectable phenotypic change resulting from the GFP tagging. We analysed the expression and subcellular location of these GFP-tagged proteins by live cell imaging at various stages of the life cycle. Taken together with the previously published results for kinesin-5, $-8 \mathrm{~B}$ and $-8 \mathrm{X}$ (Zeeshan et al., 2020a; Zeeshan et al., 2019a; Zeeshan et al., 2019b), we found that the nine kinesins have a diverse pattern of expression, with distinct subcellular locations including the mitotic spindle, axonemes, the surface pellicle and a polar distribution at various stages of the parasite life cycle (Fig. 1B). The diffuse distribution of kinesin4GFP expression was detected only during oocyst development (Fig. 1C). Interestingly, only two kinesins, kinesin-5 and -13, were expressed throughout the parasite life cycle, including blood stage schizogony, and were located on the mitotic spindle in both asexual and sexual stages (Fig. 1C). Kinesin-5GFP was restricted to the nucleus and not detected in mature extracellular parasites such as merozoites, male gametes and sporozoites, while kinesin-13GFP had both a nuclear and cytoplasmic location (Fig. 1C). Kinesin-8XGFP was also located on the nuclear spindle but only during the proliferative stages within the mosquito vector. Three kinesins (kinesin-8B, -15 and $-4 \mathrm{X}$ ) were expressed only during male gametogenesis with cytosolic locations (Fig. 1C), and two kinesins (kinesin-20 and -X3) were first detected in female gametocytes with a diffuse location (Fig. 1C). Their presence continues into the zygote and later stages of ookinete differentiation and sporogony with particular locations that are discussed in detail below (Fig. 1C). We also observed two kinesins at polar locations: kinesin-8X at the basal end of stage $\mathrm{V}$ to $\mathrm{VI}$ ookinetes and kinesin-13 at the apical end throughout ookinete development (Fig. 1C). Overall, the phylogenetically conserved kinesin-5 and $-8 \mathrm{X}$ are restricted to nuclear spindle and kinesin-13 is present in both nucleus and cytoplasm. The Apicomplexa-enriched kinesin-X3 and -X4 are confined to ookinete and sporozoite pellicle and flagellar axoneme, respectively.

\section{Kinesin-5 and $-8 \mathrm{X}$ are nuclear spindle kinesins associated with the kinetochore (NDC80) that bind centromeres.}

In our previous studies we showed that the location of two kinesins, -5 and $-8 \mathrm{X}$, is associated with spindles and restricted to the nucleus during most of the life cycle stages (Zeeshan et al., 2020a; Zeeshan et al., 2019b). To further examine the spatio-temporal dynamics of these kinesins during spindle formation, chromosome segregation and axoneme biogenesis during male gametogenesis, we crossed parasite lines expressing kinesin-5-GFP and -8X-GFP with lines expressing NDC80-Cherry, a kinetochore protein in the nucleus, and kinesin-8B-Cherry, an axonemal protein in the cytoplasm, and compared protein location by live-cell imaging. Both kinesin-5 and -8X (green) were colocalised with NDC80 (red) suggesting a role in mitotic spindle function and chromosome segregation (Fig. 2A and B). On the other hand, neither kinesin-5 nor kinesin-8X showed any overlap with cytosolic kinesin-8B (red) during male gametogenesis (Fig. S2A and B) confirming their restricted location within the nuclear compartment. 
Kinetochores are multiprotein complexes assembled at the centromere of each chromosome, which mediate chromosome attachment to spindle MTs. Because kinesin5 and $-8 X$ showed colocalization with kinetochore protein NDC80, we analysed further the binding of these proteins at the centromere DNA. We performed ChIP-seq experiments with parasites undergoing gametogenesis (6 min post-activation [mpa]), using kinesin-5GFP and -8XGFP tagged parasites and GFP-specific antibodies. Strong ChIP-seq peaks for each chromosome were observed with these kinesins, indicating their binding sites. Binding was restricted to a region close to the previously annotated centromeres of all 14 chromosomes (Iwanaga et al., 2012) and identical to those identified for Plasmodium Condensin and NDC80 studies (Pandey et al., 2020; Zeeshan et al., 2020b) (Fig. 2C). Together, live cell imaging and ChIP-seq analysis support the notion that kinesin- 5 and $-8 \mathrm{X}$ associate with kinetochores assembled at centromeres.

\section{Apicomplexa-enriched kinesins have discrete locations during pellicle formation (-X3) and axoneme assembly (-X4) during sexual development.}

Previous bioinformatic analysis identified two divergent Plasmodium kinesins (kinesin-X3 and -X4) (Wickstead et al., 2010; Zeeshan et al., 2019b); one of them (kinesin-X3) is restricted to the phylum Apicomplexa (Zeeshan et al., 2019b). Kinesin-X4 is also restricted to Apicomplexa except that it is also in the starlet sea anemone Nematostella vectensis (Wickstead et al., 2010). Parasitic organisms of Apicomplexa are characterised by a specialised apical structural complex that coordinates the interaction with and penetration of host cells, and have a surface pellicle comprised of the plasma membrane and an underlying layer of fused flattened membrane vesicles of the inner membrane complex (IMC) with associated MTs (Gould et al., 2008; Kono et al., 2013). To examine whether the kinesins are associated with these apicomplexan features, localisation by live cell imaging was performed. Kinesin-X3 and $-X 4$ had a stage-specific expression during sexual development with a distinct location (Fig. 1C). During zygote to ookinete differentiation, kinesin-X3 expression was restricted to one side of the cell in the early stages of development (stage I-III), suggesting an involvement in pellicle formation (Fig. 3A). In later stages (stage IV-VI), the kinesin-X3 location became more distinct around the periphery of the ookinete. Monoclonal antibody 13.1 conjugated with cy3 (red), which recognises the P28 protein on the surface of zygote and ookinete stages, stained these stages and colocalised with kinesin-X3 (green) (Fig. 3A), although kinesin-X3 was not present at the apical and basal ends of the developing ookinete (Fig. 3A). This suggests that kinesin-X3 is restricted to pellicle formation during ookinete and sporozoite stages in the mosquito.

Using live-cell imaging during male gametogenesis, the expression and location of kinesin-X4 (green) was compared with that of axonemal protein kinesin-8B (red) located on basal bodies and axonemes (Zeeshan et al., 2019a). Kinesin-X4 showed a diffuse cytosolic distribution during early stages of male gametogenesis (1-3 mpa) but no strong signal on the basal body tetrads labelled with kinesin-8B (red) (Fig. 3B). However, at 4-6 mpa the kinesin- $X 4$ signal distribution changed to resemble linear structures, which was maintained in the later stages (8-10 mpa) and showed co-localization with kinesin-8B (Fig. 3B). These data suggest that kinesin-X4 is located on axonemes together with kinesin-8B during flagella formation in Plasmodium spp.

Genome-wide functional screen reveals that eight out of nine kinesins are required only for parasite transmission and not for blood stage proliferation.

Previously we described the functional roles during mosquito stages of three kinesins, -5 , $-8 \mathrm{~B}$ and $-8 \mathrm{X}$; proteins that were not essential during blood stage development (Zeeshan 
et al., 2020a; Zeeshan et al., 2019a; Zeeshan et al., 2019b). To study the function of the remaining six kinesins throughout the life cycle, each gene was deleted from $P$. berghei using a double crossover homologous recombination strategy as described previously (Tewari et al., 2010) (Fig. S3A). Successful integration of the targeting constructs at each gene locus was confirmed by diagnostic PCR (Fig. S3B), except that kinesin-13 could not be deleted. PCR analysis of knockout parasites confirmed the complete deletion of these kinesin genes (Fig. S3B), indicating that they are not essential during the asexual blood stage. kinesin-13, which could not be deleted despite several attempts, likely has an essential role during the asexual blood stage. A recent functional profiling of the $P$. berghei genome (Bushell et al., 2017) also supports an essential role for kinesin-13 during the blood stage. This study found that five kinesins (kinesin-4, -8B, $8 \mathrm{X},-20$ and $-\mathrm{X} 4)$ are not essential for blood stage growth but provided no data for kinesin-5, -15 and $-X 3$ (Bushell et al., 2017).

Phenotypic analyses of these kinesin-knockout parasites, in comparison with the parental parasite (WTGFP), were carried out at various stages of the life cycle: in asexual blood stages, during male gametogenesis and the formation of exflagellation centres, during ookinete formation, in the number and size of oocysts, for the formation of sporozoites in oocysts and their migration to salivary glands, and for parasite transmission to the vertebrate host (Fig. 4A). Taken together with previously published studies on kinesin-5, $-8 \mathrm{~B}$ and $-8 \mathrm{X}$, only two knockout parasite lines ( $\triangle$ kinesin- $8 B$ and $\Delta$ kinesin-15) showed a defect in the formation of male gametes (Fig. 4B). $\Delta$ kinesin- $8 B$ parasites produced no male gametes, as shown previously (Depoix et al., 2020; Zeeshan et al., 2019a), while there was a significant decrease in male gamete formation in $\Delta$ kinesin-15 parasites (Fig. 4B). Next, we analysed the zygote to ookinete transition (round to banana-shaped cells) after $24 \mathrm{~h}$ following gametocyte activation. Three parasite lines ( $\Delta$ kinesin-8B, $\Delta$ kinesin-15 and $\Delta$ kinesin-20) produced no or reduced numbers of ookinetes (Fig. 4C). $\Delta$ kinesin-8B parasites produced no ookinetes, as expected because there were no male gametes to fertilise the female gametes (Fig. 4C) (Zeeshan et al., 2019a). $\Delta$ kinesin-15 parasites produced significantly fewer male gametes, which would be expected to result in fewer ookinetes compared to WTGFP parasites (Fig. 4C). In contrast, $\Delta$ kinesin-20 parasites exflagellated normally and therefore loss of this kinesin must have a direct effect on ookinete formation (Fig. 4C).

To assess the effect of kinesin gene deletions on oocyst development and infective sporozoite formation, 40-50 Anopheles stephensi mosquitoes were fed on mice infected with individual kinesin-knockout lines, and parasite development was examined. First, GFP-positive oocysts on the mosquito gut wall were counted at 7-, 14and 21-days post-infection (dpi). Three out of eight kinesin-knockout lines showed defects in oocyst production; $\Delta$ kinesin-8B parasites produced no oocysts as shown previously (Zeeshan et al., 2019a), while there was a significant reduction in $\Delta$ kinesin-15 and $\Delta$ kinesin-20 oocysts compared to WTGFP oocysts at $7 \mathrm{dpi}$ and a further reduction by 14 and $21 \mathrm{dpi}$ (Fig. 4D). The adverse effects on ookinete production rather than a direct effect on oocyst development could explain this observation. Although there was no significant difference in the number of oocysts of other kinesin gene knockouts compared to WTGFP at $7 \mathrm{dpi}$, a significant reduction was observed for the $\Delta$ kinesin$8 X$ line at $14 \mathrm{dpi}$, which became more evident by $21 \mathrm{dpi}$ (Fig. 4D). Oocyst size was not affected in most of the lines that produced them, the only exception was $\Delta$ kinesin- $8 X$ oocysts, which were approximately half the size of WTGFP oocysts at $14 \mathrm{dpi}$, and even smaller by 21 dpi (Fig. 4E). It would appear that kinesis-8X is the only kinesin that directly affects oocyst development. Four out of eight kinesin-knockout lines produced no or defective sporozoites; $\Delta$ kinesin- $8 B$ and $\Delta k$ kinesin- $8 X$ produced no sporozoites, as 
reported earlier (Zeeshan et al., 2019a; Zeeshan et al., 2019b), while $\Delta$ kinesin-15 and $\Delta$ kinesin-20 lines had significantly reduced sporozoite numbers compared to control parental parasites (Fig. 4F). These defects were mirrored in the salivary glands: for the $\Delta k$ inesin-8B and $\Delta k$ inesin- $8 X$ lines no sporozoites were detected, as reported earlier (Zeeshan et al., 2019a; Zeeshan et al., 2019b), while $\Delta$ kinesin-15 and $\Delta$ kinesin-20 lines had a significantly reduced number. The $\Delta k i n e s i n-5$ parasite produced significantly fewer infective salivary gland sporozoites (Fig. $\mathbf{4 G}$ ) as reported previously (Zeeshan et al., 2020a). However, although several kinesin gene-knockout lines exhibited defects in sporozoite production and reduced salivary gland infection, these sporozoites were still infectious to the mammalian host as observed with successful infection of new hosts in mosquito bite back experiments (Fig. 4H). In summary, for most of the kinesin gene-knockout $P$. berghei lines, there were clear developmental defects at specific stages of the life cycle within the mosquito vector.

\section{Kinesin-20-GFP location reveals a ring-like structure during ookinete differentiation, and deletion of the kinesin-20 gene affects ookinete morphology and motility.}

In the initial phenotypic screen described above, the $\Delta$ kinesin-20 parasite did not produce normal ookinetes (Fig. 4C) but did produce a few oocysts (Fig. 4D), so we undertook a more in-depth analysis to investigate this further. First, we analysed the spatiotemporal profile of kinesin-20GFP expression during zygote to ookinete differentiation, using P28 as a cell surface marker. Live cell imaging showed a diffuse distribution of kinesin-20GFP in the female gametocyte and zygote (Fig. 1C, 5A). Subsequently, the intensity of kinesin-20GFP increased in the developing apical protuberance from the main cell body, and at later stages, especially at stage-II and III that are about 6-8 $\mathrm{h}$ after gametocyte activation (Fig. 5A). The protein appeared like a ring restricted to the junction of the main cell body and the protrusion that is characteristic of developing ookinetes during stage II to stage V, and then largely disappeared in mature ookinetes (stage $\mathrm{VI}$ ) with a largely diffuse distribution similar to that of the zygote stage (Fig. 5A).

Next, we examined the $\Delta$ kinesin-20 parasites to ascertain whether the zygote was formed and at what stage parasite development was blocked. The $\Delta k$ kinesin-20 parasite developed a short protuberance in stage-II similar to that of the WTGFP control (Fig. 5B), but this protrusion developed into a bulbous structure rather than the characteristic banana-shaped ookinete (Fig. 5B) and remained like this $24 \mathrm{~h}$ later when the WTGFP ookinete had fully differentiated into the banana-shaped structure (Fig. 5B and C). Since the mature wildtype ookinete is a motile and invasive stage, we investigated the motility of the $\Delta$ kinesin-20 bulbous ookinete using a Matrigel substrate, as described previously (Volkmann et al., 2012; Zeeshan et al., 2019b). There was a significant decrease in the motility of $\Delta$ kinesin-20 ookinetes compared with the WTGFP ookinetes that showed normal gliding motility (Fig. 5D and E; movies S1 and S2).

Although $\Delta$ kinesin-20 parasites made no morphologically normal and motile ookinetes, nevertheless they formed a few oocysts that produced sporozoites (Fig. 4F). This would point to the mutation specifically affecting motility but not invasion of the mosquito gut. It is possible that a few immotile ookinetes may contact the mosquito gut wall during gut turbulence and these are able to invade and initiate oocyst formation. The $\Delta$ kinesin-20 sporozoites were morphologically similar to WTGFP parasites, so we examined their motility on Matrigel as described previously (Volkmann et al., 2012; Wall et al., 2019). The motility of $\Delta$ kinesin-20 sporozoites was similar to that of WTGFP 
sporozoites (Fig. 5F and G; movies S3 and S4), suggesting that the defect in $\Delta$ kinesin20 parasite shape and motility is limited to ookinete development.

\section{qRTPCR and RNA-seq analysis of $\Delta$ kinesin-20 parasites showed the modulation of a limited number of genes.}

In order to examine whether the expression of other kinesin genes is mis-regulated in $\Delta$ kinesin-20 parasites, we performed qRTPCR analysis of them. None of the other kinesin genes showed any significant change in expression in $\Delta$ kinesin-20 parasites compared with WTGFP parasites (Fig. 5H).

To determine genome-wide changes in transcription we performed RNA-seq analysis of the $\Delta$ kinesin-20 gametocytes at $2 \mathrm{~h}$ post activation. The kinesin- 20 deletion in the $\Delta$ kinesin-20 strain was confirmed, since no significant reads mapped to this gene locus (Fig. 5l). The volcano plot shows that only one gene was upregulated, and 16 genes were down regulated (Fig. 5J, Table S1A). Most of the differentially regulated genes belong to pir and fam gene clusters localized in the telomeric and sub-telomeric regions. Two of the most downregulated genes (PBANKA_1465700 and PBANKA_0200600) in $\Delta$ kinesin-20 gametocytes belong to the fam gene cluster and were described as female gametocyte specific genes in a recent study (Reid et al., 2018). This suggests a link of kinesin-20 with female gametocyte development and function.

\section{Ultrastructure analysis of $\Delta$ kinesin-20 ookinetes reveals disorganised subpellicular microtubules.}

The defective size and shape of $\Delta k$ inesin-20 parasites during ookinete differentiation led us to perform high resolution and ultrastructure analysis of the bulbous ookinetes using ultrastructure expansion- and electron microscopy. With expansion microscopy using MT-specific antibody, we observed a marked reduction in microtubule length (Fig. 6A): the length of MTs in $\Delta$ kinesin-20 bulbous ookinetes was decreased by approximately $40 \%$ compared to those of WTGFP parasites, a difference which also reflected a shortening of the overall length of the ookinete (fig. S4). This difference was confirmed by electron microscopy observations (Fig. 6B).

In an ultrastructural comparison of WTGFP and $\Delta$ kinesin-20 ookinetes, the most obvious difference was the shape of the cell. In longitudinal section, the WTGFP ookinetes were elongated with a crescentic outline (Fig. 6Ba), but in contrast, longitudinal sections of the $\Delta k$ inesin-20 ookinetes were less elongated and had a more bulbous appearance (Fig. 6Bb, c). In WTGFP parasites, the distribution of subcellular organelles appeared ordered with most micronemes in the apical cytoplasm, a more centrally located crystalline body and a posterior nucleus (Fig. 6Ba). In contrast, early $\Delta$ kinesin-20 ookinetes had a large central nucleus with a few dense granules but lacked both micronemes and a crystalline body (Fig. 6Bb). Others that appeared more mature, possessed similar organelles (micronemes, crystalline body, nucleus) to those of the WTGFP, but differed from the control in having more randomly distributed micronemes (Fig. 6Bc).

Due to the differences in cell shape, the apical complex and pellicle were examined in detail. When the apical complex was examined in longitudinal (Fig. 6Bd, e) and cross (Fig. 6Bf, g) section, the complex nature of the structure was revealed. Interestingly, WTGFP and $\Delta$ kinesin-20 ookinetes displayed an identical sub-structure (Fig. 6Bd-e and $\mathrm{f}-\mathrm{g}$ ). In longitudinal sections of the central apex region, three conoidal rings could be identified underneath the plasma membrane. A unique sub-structure of the ookinete is the apical collar, which represents a cone-like structure embedded 
415 between the microtubules and IMC of the pellicle (Koreny et al., 2021). The outer region of the collar is electron dense and appears to be fused to the IMC, which is interrupted at the apical end to form apical polar ring 1 (Fig. 6Bd-g). The inner aspect of the collar is more electron lucent and in close contact with the sub-pellicular MTs (Fig. 6Bd-g). The apical ends of the MTs are attached to a ring forming apical polar ring 2 (Fig. 6Bd, e). For a detailed review of the apical complex see Koreny et al (Koreny et al., 2021). Approximately 50 sub-pellicular MTs emanate from polar ring 2 and run longitudinally beneath the collar (Fig. 6Bf, g) and then beneath the IMC of the pellicle (Fig. 6Bh, i). In the region of the collar, MTs were evenly distributed in both WTGFP and $\Delta$ kinesin-20 parasites (Fig. 6Bf, g), however, in more posterior sections, while there continued to be an even distribution of MTs in close contact with the IMC in the WTGFP (Fig. 6Bh) ookinete, in the $\Delta$ kinesin-20 parasite there were areas where there was uneven distribution, clumping and detachment of the MT from IMC (Fig. 6Bi).

\section{Kinesin-13 associates with kinetochore marker NDC80 at all proliferative stages of the life cycle and its knockdown affects male gamete formation.}

Since kinesin-13 was the only kinesin gene that was essential for blood stage schizogony and could not be disrupted in our genome-wide screen (Fig. 4), we performed a detailed analysis of kinesin-13. We observed both a diffuse cytoplasmic distribution and a distinct nuclear pattern of kinesin-13GFP during all proliferative stages as shown in Fig. 1. First, we performed live cell co-imaging of kinesin-13GFP and the NDC80-cherry kinetochore marker after crossing the two transgenic parasite lines, to observe kinesin13-GFP dynamics during chromosome segregation in various developmental stages. There was colocalization of kinesin-13 and NDC80 at all proliferative stages (Fig. 7), for example during the schizogony and sporogony endomitotic stages (Fig. 7A, B). In the sexual cells, during the rapid mitosis of male gametogenesis there was partial colocalization of kinesin-13 and NDC80, but a substantial amount of kinesin-13 was also located in the cytosolic compartment (Fig. 7C). In the meiotic stage during ookinete development there was clear colocalization of kinesin-13 and NDC80 (Fig. 7D). At the start of meiosis (2 $\mathrm{h}$ after zygote formation) there was one strong nuclear focus and at the end of ookinete formation there were three to four co-localised foci (Fig. 7D). Further analysis by fixed immunofluorescence microscopy of kinesin-13 (green) in the male gamete revealed a co-localization with tubulin (red) (Fig. S5A). To further examine the location of kinesin-13, we used ultrastructure expansion microscopy to examine gametocytes activated for $15 \mathrm{~min}$, and then compared its location with that of tubulins. Kinesin-13 (green) was observed to colocalized with $\alpha / \beta$ tubulin (magenta) suggesting a location on axonemes and spindles (Fig. S5B).

Since, kinesin-13 is essential for the asexual blood stage, and the gene could not be deleted, we applied two conditional genetic knockdown strategies to evaluate its role at other proliferative stages within mosquito vector. First, we used an auxin-inducible degron (AID) system to try and study the effect of rapid kinesin-13 degradation in gametocytes. We tagged the endogenous kinesin-13 gene with an AID/HA epitope tag (Fig. S6A) to degrade the fusion protein in presence of auxin (Philip and Waters, 2015), and successfully generated kinesin-13-AID parasite lines as shown by integration PCR (Fig. S6B) but could not deplete kinesin-13 protein by auxin treatment (Fig. S6C). Next, we used a promotor trap double homologous recombination (PTD) approach, inserting the clag promotor at the 5' end of kinesin-13, and generated the conditional knockdown parasite: Pclagkinesin-13 (kinesin-13PTD) (Fig. S6D). The clag (cytoadherence-linked asexual gene) is highly expressed in asexual blood stages, but largely silent during 
stages of sexual differentiation, including gametocytes and ookinetes (Sebastian et al., 2012). The successful insertion was confirmed by diagnostic PCR (Fig. S6E) and qRT PCR showed a significant downregulation of kinesin-13 gene transcripts in kinesin13PTD gametocytes, when compared to WTGFP gametocytes (Fig. 8A).

The phenotype of the kinesin-13PTD modification was examined during various stages of the parasite life cycle. Parasite proliferation in asexual blood stages was not affected, but during male gametogenesis, exflagellation was markedly reduced and very few male gametes were produced by kinesin-13PTD parasites compared to WTGFP parasites (Fig. 8B). Zygote development and ookinete differentiation were severely affected, and no clear banana-shaped ookinetes were observed (Fig. 8C). Subsequent stages in the mosquito were also affected significantly and no oocyst or sporozoite formation was observed (Fig. 8D), and as a consequence no transmission of kinesin13PTD parasites occurred, as shown by mosquito bite back experiments (Fig. 8E).

\section{Global transcriptomic analysis of kinesin-13PTD parasites shows mis-regulation of transcripts for gene clusters involved in axoneme assembly and chromosome dynamics.}

To examine the transcript level of other kinesins in kinesin-13PTD gametocytes, we performed $\mathrm{qPCR}$ for all the nine kinesins and found that some, like kinesin-8B, $-8 \mathrm{X},-15$ and -20 , were downregulated (Fig. 8F).

Since transcripts of other kinesins were affected, global transcription was investigated by RNA-seq analysis of kinesin-13PTD gametocytes at 0 and $15 \mathrm{mpa}$, representing times point before the start of male gametogenesis and just after male gamete formation (exflagellation), respectively. Kinesin-13 downregulation in kinesin13PTD gametocytes, relative to WTGFP gametocytes, was confirmed by RNA-seq analysis, showing the lack of reads for this locus (Fig. 8G). In addition to reduced kinesin-13 expression, 34 other genes were significantly downregulated and the expression of 152 genes was significantly upregulated in kinesin-13PTD gametocytes before activation (at $0 \mathrm{mpa}$ ) (Fig. $8 \mathrm{H}$; Table S1B). Similarly, the expression of 22 genes was significantly downregulated and the expression of 329 genes was significantly upregulated in kinesin-13PTD gametocytes after 15 min activation (Fig. 8I; Table S1C). Bioinformatic analysis of these differentially regulated genes revealed two important clusters of genes that were affected, including those coding for proteins involved in axoneme assembly, glideosome assembly and chromosome dynamics (Fig. 8J).

\section{High resolution ultrastructure analysis of kinesin-13PTD parasites identified defects in spindle assembly and axoneme MT of gametocytes, and the subpellicular MT of ookinetes.}

Phenotypic analysis of kinesin-13PTD parasites revealed defects in male gamete and ookinete formation, and therefore we performed comparative high resolution image analysis of kinesin-13PTD and WTGFP gametocytes and ookinetes. Ultrastructure expansion microscopy revealed that both spindle and axoneme MTs were disorganised, and no clear flagella were visible at 4 to 5 or 15 min after male gametocyte activation, respectively (Fig. 9A). Disorganised MT were also observed in the defective kinesin13PTD zygotes/ookinetes in comparison to the corresponding WTGFP parasites (Fig. 9A).

These data were substantiated by electron microscopy analysis of male gametocytes activated for 6 or $15 \mathrm{~min}$ (Fig. 9B). The electron micrographs showed that in both WTGFP and kinesin-13PTD parasites at $6 \mathrm{mpa}$, most male gametocytes were at early stage of development, with a spherical appearance and a large central nucleus 
515 (Fig. 9Ba, C). In WTGFP gametocytes, a number of nuclear poles were observed while 516 the cytoplasm contained a number of normal 9+2 axonemes, and some abnormal axonemes (Fig. 9Ba, b). Although in the kinesin-13PTD gametocytes few nuclear poles were observed (Fig. 9Bc), the major difference was in the cytoplasm, where there were collections of free single and double MTs plus a number partially organized into axonemes-like structures (Fig. 9Bd) while 9+2 axonemes were very rare. At $15 \mathrm{mpa}$, in the WTGFP samples, a number of late stages were observed with evidence of exflagellation and protruding microgametes (Fig. 9Be). A number of free male gametes complete with nucleus and flagellum was observed (Fig. 9Bf, g). In contrast, in the kinesin-13PTD parasites, the majority of male gametocytes were still at an early stage, and in the few at a later stage, the nucleus displayed clumps of chromatin (Fig. 9Bh) with a few examples of protrusions of MTs from the plasma membrane (Fig. $9 \mathrm{Bi}$ ), but with no evidence of flagella formation or free male gametes. 


\section{Discussion}

Plasmodium spp. have a complex life cycle involving two hosts. They invade tissues and cells in diverse environments and have evolved a series of cellular shapes and sizes, with several distinct morphological forms with cellular polarity and gliding motility for invasion, and cellular proliferation underpinned with an atypical mode of cell division (Gubbels et al., 2020; Sinden, 1991; Zeeshan et al., 2020b). Many of these processes are governed by MTs and MT-based motor proteins like kinesins (Zeeshan et al., 2019b). In many organisms including Plasmodium spp., MTs form different structural frameworks such as the spindle assembly during cell division, the axonemes of cilia and flagella, and a cytoskeletal scaffold to establish and maintain cell polarity, cell shape, intracellular transport, and cell motility (Morrissette and Sibley, 2002; Spreng et al., 2019). Kinesins regulate the organisation and function of MTs, using them as a track for movement or regulating their dynamics during cellular processes (Verhey and Hammond, 2009; Vicente and Wordeman, 2015). Plasmodium spp. are evolutionarily divergent unicellular eukaryotes with genomes that encodes nine kinesins including two that are Apicomplexa-enriched, and which lack three classical kinesins (kinesin-1, -2 and -3) normally important in intracellular transport (Wickstead et al., 2010; Zeeshan et al., 2019b). In the present study we performed a systematic comparative analysis of the timing of expression and subcellular location of all kinesins, and their roles in parasite proliferation, polarity and transmission throughout the life cycle. This comprehensive study also included the three previously described kinesins (Kinesin-5, -8B and -8X). (Zeeshan et al., 2020a; Zeeshan et al., 2019a; Zeeshan et al., 2019b).

The expression and subcellular location of each kinesin provides important information about their potential role. Three of the most evolutionarily conserved kinesins (kinesin-5, -8X and -13) are abundant throughout the life cycle with a similar location on spindles and associated with the kinetochore protein NDC80, consistent with a role in spindle dynamics during endomitosis and meiosis, as shown in our recent studies (Zeeshan et al., 2020a; Zeeshan et al., 2019b). Kinesin-13 is the most widely expressed of these motors in all proliferative and invasive stages, with diverse cytoplasmic locations including axonemes in male gametocytes and at the apical end of the differentiating ookinete in addition to its association with the nuclear spindle apparatus. A similar diverse set of kinesin-13 locations has been reported in other eukaryotes (Ali et al., 2017; Ems-McClung and Walczak, 2010; Manning et al., 2007), highlighting its importance for various MT-associated biological processes in Plasmodium spp..

Male gametogenesis in Plasmodium spp. is a rapid process that includes three rounds of genome replication with successive spindle formation and cytoplasmic axoneme assembly before karyokinesis and cytokinesis, and all completed within 12-15 min of gametocyte activation (Sinden et al., 1978). The cytoplasmic location of three male gametocyte-specific kinesins (kinesin-8B, -15 and -X4) and kinesin-13, highlights the importance of these kinesins for rapid axoneme assembly and flagellum formation in Plasmodium spp., especially kinesin-X4. This motor is phylogenetically largely restricted to Apicomplexa and displays a male gametocyte-specific expression with an axonemal location. Kinesin-X4 colocalizes with kinesin-8B but is not essential like kinesin-8B, suggesting that it might be complementing the role of kinesin-8B (Zeeshan et al., 2019a). In addition to Apicomplexa, kinesin-X4 is found in the starlet sea anemone Nematostella vectensis, where its function is also unknown (Wickstead et al., 2010). The axonemes are assembled directly in the male gametocyte cytoplasm and, thus, there is no requirement for transport of building materials by the intraflagellar transport (IFT) mechanisms common in many other eukaryotes (Briggs et al., 2004; Mirvis et al., 2018; Sinden et al., 2010). Plasmodium spp. male gametes have a very simple structure, 
consisting of an electron dense basal body that lacks the classical nine triplet MTs $(9+0)$ connected to an axoneme with a typical 9+2 MT organization associated with dynein, with an adjacent elongated nucleus all enclosed within the plasma membrane (Okamoto et al., 2009; Sinden et al., 1978). In a previous Plasmodium berghei male gamete proteome study, three kinesins (kinesin-8B, -13 and -15 ) were identified and proposed to have an important role in axoneme assembly (Talman et al., 2014). In a recent study on the regulation of $P$. berghei male gametogenesis, a large number of phospho-regulated proteins have motor activity, and they include most of these kinesins (Invergo et al., 2017). The expression of six kinesins (kinesin-5, -8B, -8X, -13, -15, and -X4) in gametocytes and their location in either nucleus (kinesin-5 and $-8 X$ ), cytoplasm (kinesin$8 \mathrm{~B},-15$, and $-\mathrm{X} 4$ ) or both (kinesin-13) suggest the importance of these kinesins in male gametogenesis and thus parasite transmission.

The restricted location of kinesin-X3 at the pellicle of ookinetes, except for the apical and basal ends of the cell, suggests an involvement in subpellicular MT/IMC interactions. The absence of kinesin- $\mathrm{X} 3$ from the apical end highlights that it is not a part of the conoidal complex (Bertiaux et al., 2021; Koreny et al., 2021). Kinesin-20 has a ring-like location at the junction between the protrusion and the main cell body of the developing ookinete, suggesting a role in formation of the IMC/sub-pellicular microtubule complex and defining the size and shape of the cell, and then disappears from the mature cell. Plasmodium Myosin F has a similar location in early stages of ookinete development (Wall et al., 2019) suggesting the existence of an actomyosin contractile ring that might be regulated by kinesin-20.

Genetic analysis revealed that most of the kinesins (8 out of 9 ) have their most important roles in transmission stages within the mosquito, where there are substantial changes in cell size, morphology, and invasive properties, which may be regulated by MTs and associated proteins. For example, the results of our ultrastructural analysis of $\Delta$ kinesin-20 parasites show that loss of this kinesin affects the development of ookinete shape and size. MTs length, number and association with the IMC are crucial to determine the size, shape and motility of certain Plasmodium spp. stages (Spreng et al., 2019). We show that kinesin-20 regulates the length and arrangement of subpellicular MTs of developing ookinetes. Subpellicular MTs along with IMC proteins maintain ookinete polarity and morphology in Plasmodium spp. (Kono et al., 2013). IMC1bdeficient ookinetes display abnormal cell shape and reduced gliding motility (Tremp et al., 2008) similar to the properties of $\Delta$ kinesin-20, and a similar phenotype was observed in a recent study showing that palmitoylation of IMC subcompartment proteins (ISPs) regulates the apical subpellicular MT network in ookinetes and affects their elongation (Wang et al., 2020). ISPs also maintain the polar location of guanylate cyclase beta (GC $\beta$ )/CDC50A complex at the IMC, essential for ookinete gliding (Gao et al., 2018). PPKL-deficient parasites have a defective MT organisation and abnormal shaped ookinetes (Guttery et al., 2012), but the $\Delta$ kinesin-20 phenotype is slightly different, with no defect in the apical ring, which serves as an MT organising centre for subpellicular MTs, and similar to what was found for phosphodiesterase- $\delta(p d e \delta)$ deficient ookinetes, which lack this enzyme involved in cyclic GMP signaling (Moon et al., 2009). The kinesin-20GFP location suggests there is a ring-like structure at the junction of the protrusion and cell body, which defines the developing ookinete shape and diameter, while apical polarity guides ookinete size and differentiation. An actomyosin contractile ring is present in elongation of embryonic cells of Ciona intestinalis, a primitive chordate (Sehring et al., 2014). The assembly and organization of an actomyosin contractile ring during cytokinesis is highly dynamic and contains, in addition to actin and myosin, other proteins that regulate actin nucleation, cross-linking and myosin activity (Mangione and 
Gould, 2019; Vavylonis et al., 2008). In Plasmodium spp. and other members of Apicomplexa, a similar contractile ring has been reported as required for cytokinesis (Hammarton, 2019; Morano and Dvorin, 2021) but an involvement in cell elongation is unknown. Kinesin-20 could be a protein that regulates contractile ring function in cell elongation during ookinete development.

The in-depth structural analysis of kinesin-13PTD parasites reveals the importance of kinesin-13 in regulating MT organisation in sexual stages in the mosquito. Kinesin-13s are MT depolymerising kinesins, playing essential roles in spindle MT dynamics, kinetochore-MT attachment, and chromosome segregation (Ali et al., 2017; Ems-McClung and Walczak, 2010; Manning et al., 2007). Plasmodium falciparum kinesin-13 has also been shown to exhibit MT depolymerisation activity in vitro (Moores et al., 2002). Kinesin-13 homologs are present in most eukaryotes possessing cilia or flagella (Wickstead and Gull, 2006) and regulate the length of these structures (Chan and Ersfeld, 2010; Piao et al., 2009; Vasudevan et al., 2015). The knockdown of Plasmodium berghei kinesin-13 resulted in defects in the organisation of both spindle and axonemal MTs, thus arresting nuclear division and axoneme assembly during male gametogenesis. A similar phenotype was observed for the kinesin-8B gene knockout, which shows defective basal body formation and axoneme assembly during male gametogenesis, but nuclear division was normal (Zeeshan et al., 2019a). Knockout of the gene for another basal body protein, SAS6, resulted in a similar phenotype with defective basal body formation and axoneme assembly but no effect on nuclear division (Marques et al., 2015). Disruption of the gene for PF16, an armadillo-repeat protein of Plasmodium spp., produces a similar phenotype, with an effect on axonemal central MT assembly and male gamete motility and fertility (Straschil et al., 2010). We found a cdc2-related kinase (CRK5) that is important for nuclear spindle formation but has no effect on axoneme assembly during male gametogenesis (Balestra et al., 2020). Previous studies have shown a similar phenotype for CDPK4, SRPK and MAP2 gene disruptions, where either the early or late stages of exflagellation are impaired but axoneme assembly is not (Fang et al., 2017; Tewari et al., 2005; Tewari et al., 2010). In another primitive eukaryote, Giardia intestinalis, kinesin-13 regulates MT dynamics during both flagellar assembly and nuclear division (Dawson et al., 2007).

Since kinesin-13 was not completely depleted, a few male gametes were produced that fertilised female gametes to form zygotes. However, these zygotes did not transform to ookinetes and produced only abnormal forms or ones arrested at the undifferentiated (retort) stage. Ultrastructure analysis of these retorts revealed a loss of polarity and disorganisation of the subpellicular MTs, consistent with the additional polar localization of kinesin-13GFP in zygotes and during ookinete development. This phenotype of kinesin-13 depletion is different from that of the kinesin-20 knockout (described above), where the apical polarity was not affected. A similar phenotype has been observed following the knockdown of two $P$. berghei phosphatases, PPKL and PP1, where apical polarity was lost, affecting ookinete differentiation (Guttery et al., 2012; Zeeshan et al., 2021).

Global transcriptomic analysis supported these findings with kinesin-13, as the expression of several genes involved in chromosome segregation, axoneme biogenesis, IMC/glideosome formation and other biological processes were modulated in kinesin13PTD parasites. For example, differentially expressed genes like CRK5, SRPK, CDPK4 are involved in chromosome segregation during male gametogenesis (Fang et al., 2017; Tewari et al., 2005; Tewari et al., 2010). Kinesin-8B, kinesin-X4, dynein and radial spoke proteins are involved in axoneme assembly, male gamete formation and fertility (Andreadaki et al., 2020; Talman et al., 2014; Zeeshan et al., 2019a). Differential 
expression of genes like IMCs and GAPs indicates the additional role of kinesin-13 in glideosome formation and motility. Most of these changes in gene expression are obvious at 15 min post activation of gametocytes prior to gamete formation and fertilization. Translation repression in Plasmodium spp. is released after fertilization, allowing the stored transcripts in female gametocytes to be translated to form proteins essential for zygote development and ookinete invasion (Mair et al., 2006). Differential expression, mainly upregulation of these genes in kinesin-13PTD parasites, suggests a compensatory role during male gametogenesis and ookinete formation. Overall, these global transcriptomic data for kinesin-13PTD parasites are consistent with the profound phenotypic changes observed during male gametogenesis and ookinete formation.

In conclusion, the nine $P$. berghei kinesins show a diverse pattern of expression and subcellular location at various stages of the parasite life cycle. Genetic and phenotypic analyses indicate that most kinesins have their most important roles in mosquito stages, except kinesin-13 which is also essential for asexual blood stages (Fig. 10). Kinesin-20 and kinesin-13 have roles in MT dynamics during proliferation, polarity formation, and transmission of the parasite. It will be interesting in the future to look for the interacting partners of these kinesins, to understand their mechanisms of action during these biological processes. This comprehensive study provides knowledge and understanding of the important roles of kinesins in various cellular processes at different stages of the life cycle of this evolutionarily divergent eukaryotic pathogen. This information may be useful to exploit kinesins as promising targets for therapeutic intervention against malaria. 


\section{Materials and Methods}

\section{Ethics statement}

The animal work passed an ethical review process and was approved by the United Kingdom Home Office. Work was carried out under UK Home Office Project Licenses (30/3248 and PDD2D5182) in accordance with the UK 'Animals (Scientific Procedures) Act 1986'. Six- to eight-week-old female CD1 outbred mice from Charles River laboratories were used for all experiments.

\section{Generation of transgenic parasites and genotype analyses}

To observe the location of kinesin proteins, the C-terminus was tagged with green fluorescent protein (GFP) sequence by single crossover homologous recombination at the 3' end of the gene. To generate the GFP-tag line, a region of these genes downstream of the ATG start codon was amplified, ligated to p277 vector, and transfected as described previously (Guttery et al., 2012). The p277 vector contains the human dhfr cassette, conveying resistance to pyrimethamine. A schematic representation of the endogenous gene locus, the constructs and the recombined gene locus can be found in Fig S1A. For the parasites expressing a C-terminal GFP-tagged protein, diagnostic PCR was used with primer 1 (Int primer) and primer 3 (ol492) to confirm integration of the GFP targeting construct (Fig S1B). A list of primers used to amplify these genes can be found in Table S2.

The gene-deletion targeting vectors for kinesin genes were constructed using the pBS-DHFR plasmid, which contains polylinker sites flanking a Toxoplasma gondii dhfr/ts expression cassette conferring resistance to pyrimethamine, as described previously (Tewari et al., 2010). The $5^{\prime}$ upstream sequences from genomic DNA of kinesin genes were amplified and inserted into Apal and Hindlll restriction sites upstream of the $d h f r / t s$ cassette of pBS-DHFR. The DNA fragments amplified from the $3^{\prime}$ flanking region of kinesin genes were then inserted downstream of the $d h f r / t s$ cassette using $E c o R I$ and $X b a l$ restriction sites. The linear targeting sequence was released using Apal/Xbal. A schematic representation of the endogenous kinesin loci, the constructs and the recombined kinesin loci can be found in Fig. S3. The primers used to generate the mutant parasite lines can be found in Table S2. A diagnostic PCR was used with primer 1 (Int primer) and primer 2 (ol248) to confirm integration of the targeting construct, and primer 3 (KO1) and primer 4 (KO2) were used to confirm deletion of the kinesin genes (Fig. S3, Table S2).

To study the function of kinesin-13, we used two conditional knock down systems; an auxin inducible degron (kinesin13AID) system and a promoter exchange/trap using double homologous recombination (kinesin-13PTD). For the generation of transgenic Kinesin-13AID/HA line, library clone PbG01-2471h08 from the PlasmoGEM repository (http://plasmogem.sanger.ac.uk/) was used. Sequential recombineering and gateway steps were performed as previously described (Pfander et al., 2013; Pfander et al., 2011). Insertion of the GW cassette following gateway reaction was confirmed using primer pairs GW1 x kinesin-13 QCR1 and GW2 x kinesin-13 QCR2. The modified library inserts were then released from the plasmid backbone using Notl. The kinesin-13AID/HA targeting vector was transfected into the 615-parasite line and conditional degradation of kinesin-13-AID/HA in the non-clonal line was performed as described previously (Balestra et al., 2020). A schematic representation of the endogenous kinesin13 locus, the constructs and the recombined kinesin-13 locus can be found in Fig S6A. A diagnostic PCR was performed for kinesin-13 gene knockdown parasites as outlined in Fig. S6A. Primer pairs Kinesin-13QCR1/GW1, and Kinesin-13 QCR2/GW2 were used to 
determine successful integration of the targeting construct at the 3 ' end of the gene (Fig S6B).

The conditional knockdown construct kinesin-13PTD was derived from $P_{\text {clag }}$ (pSS367) where kinesin-13 was placed under the control of the clag gene (PBANKA_083630) promoter, as described previously (Sebastian et al., 2012). A schematic representation of the endogenous kinesin-13 locus, the constructs and the recombined kinesin-13 locus can be found in Fig. S6D. A diagnostic PCR was performed for kinesin-13 gene knockdown parasites as outlined in Fig. S6D. Primer 1 (5'-intPTD50) and Primer 2 (5'intPTD) were used to determine successful integration of the targeting construct at the 5 ' end of the gene. Primer 3 (3'-intPTclag) and Primer 4 (3'-intPTD50) were used to determine successful integration for the 3 ' end of the gene locus (Fig. S6E). All the primer sequences can be found in Table S2. $P$. berghei ANKA line 2.34 (for GFPtagging) or ANKA line 507cl1 expressing GFP (for the gene deletion and knockdown construct) parasites were transfected by electroporation (Janse et al., 2006).

\section{Parasite phenotype analyses}

Blood containing approximately 50,000 parasites of the kinesin knockout/knockdown lines was injected intraperitoneally (i.p) into mice to initiate infection. Asexual stages and gametocyte production were monitored by microscopy on Giemsa-stained thin smears. Four to five days post infection, exflagellation and ookinete conversion were examined as described previously (Guttery et al., 2012) with a Zeiss Axiolmager M2 microscope (Carl Zeiss, Inc) fitted with an AxioCam ICc1 digital camera. To analyse mosquito transmission, 30-50 Anopheles stephensi SD 500 mosquitoes were allowed to feed for 20 min on anaesthetized, infected mice whose asexual parasitaemia had reached 15\% and were carrying comparable numbers of gametocytes as determined on Giemsa stained blood films. To assess mid-gut infection, approximately 15 guts were dissected from mosquitoes on day-7 and -14 post feeding and oocysts were counted using a $63 x$ oil immersion objective. On day 21 post-feeding, another 20 mosquitoes were dissected, and their guts and salivary glands crushed separately in a loosely fitting homogenizer to release sporozoites, which were then quantified using a haemocytometer or used for imaging. Mosquito bite back experiments were performed 21 days post-feeding using naive mice, and blood smears were examined after 3-4 days.

\section{Purification of schizonts, gametocytes and ookinetes}

Blood cells obtained from infected mice (day 4 post infection) were cultured for $8 \mathrm{~h}$ and $24 \mathrm{~h}$ at $37^{\circ} \mathrm{C}$ (with rotation at $100 \mathrm{rpm}$ ) and schizonts were purified on a $60 \% \mathrm{v} / \mathrm{v}$ NycoDenz (in PBS) gradient, (NycoDenz stock solution: 27.6\% w/v NycoDenz in $5 \mathrm{mM}$ Tris- $\mathrm{HCl}$, pH 7.20, 3 mM KCl, 0.3 mM EDTA).

The purification of gametocytes was achieved by injecting parasites into phenylhydrazine treated mice (Beetsma et al., 1998) and enriched by sulfadiazine treatment after 2 days of infection. The blood was collected on day 4 after infection and gametocyte-infected cells were purified on a $48 \% \mathrm{v} / \mathrm{v}$ NycoDenz (in PBS) gradient (NycoDenz stock solution: $27.6 \% \mathrm{w} / \mathrm{v}$ NycoDenz in $5 \mathrm{mM}$ Tris- $\mathrm{HCl}, \mathrm{pH} 7.20,3 \mathrm{mM} \mathrm{KCl}, 0.3 \mathrm{mM}$ EDTA). The gametocytes were harvested from the interface and activated.

Blood cells obtained from infected mice (day 4-5 post infection) with high gametocytemia $(>20 \%)$ were cultured for $24 \mathrm{~h}$ in ookinete medium having xanthurenic acid at $20^{\circ} \mathrm{C}$. The ookinetes were pelleted at $1900 \mathrm{rpm}(500 \mathrm{~g})$, the supernatant was discarded, and the cells were resuspended in $8 \mathrm{ml}$ ookinete medium. Five $\mu \mathrm{l}$ of magnetic beads coated with 13.1 antibody (anti-P28 of ookinete) were added and incubated for $10 \mathrm{~min}$ at RT with 
continuous mixing. The tubes were placed on the magnet (Dyna rack) for 2 min and supernatant was transferred into new tubes. The beads with bound ookinetes were washed with $2 \mathrm{ml}$ of ookinete medium and used for imaging or electron microscopy.

\section{Live cell imaging}

To examine kinesin-GFP expression during erythrocyte stages, parasites growing in schizont culture medium were used for imaging at different stages (ring, trophozoite, schizont and merozoite) of development. Purified gametocytes were examined for GFP expression and cellular location at different time points $(0,1-15 \mathrm{~min})$ after activation in ookinete medium (Zeeshan et al., 2019b). Zygote and ookinete stages were analyzed throughout $24 \mathrm{~h}$ of culture. Oocysts and sporozoites were imaged using infected mosquito guts. Images were captured using a $63 x$ oil immersion objective on a Zeiss Axio Imager M2 microscope fitted with an AxioCam ICc1 digital camera.

\section{Generation of dual tagged parasite lines}

The kinesin-GFP (kinesin-5, -8X, -13 and -X4) parasites were mixed with NDC80-cherry and kinesin8B-cherry parasites in equal numbers and injected into mice. Mosquitoes were fed on mice 4 to 5 days after infection when gametocytemia was high. These mosquitoes were checked for oocyst development and sporozoite formation at day 14 and day 21 after feeding. Infected mosquitoes were then allowed to feed on naïve mice and after 4 - 5 days the mice were examined for blood stage parasitaemia by microscopy with Giemsa-stained blood smears. In this way, some parasites expressed both kinesinGFP and NDC80-cherry; and kinesin-GFP and kinesin-8B-cherry in the resultant gametocytes, and these were purified and fluorescence microscopy images were collected as described above.

\section{Ookinete and sporozoite motility assays}

Sporozoites were isolated from the salivary glands of mosquitoes infected with WTGFP and $\Delta$ kinesin-20 parasites 21 days post infection. Isolated sporozoites in RPMI 1640 containing $3 \%$ bovine serum albumin (Fisher Scientific) were pelleted (5 min, 5,000 rpm, $4^{\circ} \mathrm{C}$ ) and used for motility assay. The assay using Matrigel was performed as described previously (Volkmann et al., 2012; Zeeshan et al., 2020a). A small volume (20 $\mu$ l) of sporozoites, isolated as above for WT-GFP and $\Delta$ kinesin-20 parasites, were mixed with Matrigel (Corning). The mixture $(6 \mu \mathrm{l})$ was transferred onto a microscope slide with a cover slip and sealed with nail polish. After identifying a field containing sporozoites, time-lapse videos (one frame every $2 \mathrm{~s}$ for 100 cycles) were taken using the differential interference contrast settings with a $63 \times$ objective lens on a Zeiss Axiolmager M2 microscope fitted with an AxioCam ICc1 digital camera and analysed with the AxioVision 4.8.2 software.

For ookinete motility, $24 \mathrm{~h}$ ookinete cultures were added to an equal volume of Matrigel on ice, mixed thoroughly, dropped onto a slide, covered with a cover slip, and sealed with nail polish. The Matrigel was then allowed to set at $20^{\circ} \mathrm{C}$ for $30 \mathrm{~min}$. After identifying a field containing ookinetes, time-lapse videos were taken (one frame every 5 $s$ for 100 cycles).

\section{Fixed Immunofluorescence Assay}

The kinesin-GFP gametocytes were purified, activated in ookinete medium, fixed with 4\% paraformaldehyde (PFA, Sigma) diluted in microtubule stabilising buffer (MTSB) for 10-15 min and added to poly-L-lysine coated slides. Immunocytochemistry was performed using primary GFP-specific rabbit monoclonal antibody (mAb) (Invitrogen- 
A1122; used at 1:250) and primary mouse anti-a tubulin mAb (Sigma-T9026; used at $1: 1000$ ). Secondary antibodies were Alexa 488 conjugated anti-mouse IgG (InvitrogenA11004) and Alexa 568 conjugated anti-rabbit IgG (Invitrogen-A11034) (used at 1 in 1000). The slides were then mounted in Vectashield 19 with DAPI (Vector Labs) for fluorescence microscopy. Parasites were visualised on a Zeiss Axiolmager M2 microscope fitted with an AxioCam ICc1 digital camera.

\section{Ultrastructure expansion microscopy (U-ExM)}

Purified gametocytes were activated for 4-5 min and $15 \mathrm{~min}$; activation was stopped by adding 1X ice cold PBS. Activated gametocytes and mature ookinetes were sedimented onto $12 \mathrm{~mm}$ round Poly-D-Lysine (A3890401, Gibco) coated coverslips for $10 \mathrm{~min}$ (gametocyte procedure was performed on ice), fixed in $-20^{\circ} \mathrm{C}$ methanol for $7 \mathrm{~min}$, and then prepared for U-ExM as previously described (Bertiaux et al., 2021; Gambarotto et al., 2021). Immuno-labelling was performed using primary antibody against $\alpha$-tubulin and $\beta$-tubulin (1:200 dilution, source: Geneva antibody facility) and secondary antibody antiguinea pig Alexa 488 (1:400 dilution, source: ThermoFisher). Images were acquired on a Leica TCS SP8 microscope, image analysis was performed using Fiji-Image $\mathrm{J}$ and Leica Application Suite X (LAS X) software.

\section{Electron microscopy}

Gametocytes activated for $6 \mathrm{~min}$ and $15 \mathrm{~min}$ and ookinetes were fixed in 4\% glutaraldehyde in $0.1 \mathrm{M}$ phosphate buffer and processed for electron microscopy (Ferguson et al., 2005). Briefly, samples were post fixed in osmium tetroxide, treated en bloc with uranyl acetate, dehydrated and embedded in Spurr's epoxy resin. Thin sections were stained with uranyl acetate and lead citrate prior to examination in a JEOL JEM1400 electron microscope (JEOL Ltd, UK).

\section{RNA isolation and quantitative Real Time PCR (qRT-PCR) analyses}

RNA was isolated from purified gametocytes using an RNA purification kit (Stratagene). cDNA was synthesised using an RNA-to-cDNA kit (Applied Biosystems). Gene expression was quantified from $80 \mathrm{ng}$ of total RNA using SYBR green fast master mix kit (Applied Biosystems). All the primers were designed using primer3 (Primer-blast, NCBI). Analysis was conducted using an Applied Biosystems 7500 fast machine with the following cycling conditions: $95^{\circ} \mathrm{C}$ for $20 \mathrm{~s}$ followed by 40 cycles of $95^{\circ} \mathrm{C}$ for $3 \mathrm{~s} ; 60^{\circ} \mathrm{C}$ for $30 \mathrm{~s}$. Three technical replicates and three biological replicates were performed for each assayed gene. The hsp70 (PBANKA_081890) and arginyl-t RNA synthetase (PBANKA_143420) genes were used as endogenous control reference genes. The primers used for qPCR can be found in Table $\mathbf{S 2}$.

\section{RNA-seq analysis}

Libraries were prepared from lyophilized total RNA, first by isolating mRNA using NEBNext Poly $(A)$ mRNA Magnetic Isolation Module (NEB), then using NEBNext Ultra Directional RNA Library Prep Kit (NEB) according to the manufacturer's instructions. Libraries were amplified for a total of 12 PCR cycles (12 cycles of $\left[15 \mathrm{~s}\right.$ at $98^{\circ} \mathrm{C}, 30 \mathrm{~s}$ at $55^{\circ} \mathrm{C}, 30 \mathrm{~s}$ at $\left.62^{\circ} \mathrm{C}\right]$ ) using the KAPA HiFi HotStart Ready Mix (KAPA Biosystems). Libraries were sequenced using a NovaSeq 6000 DNA sequencer (Illumina), producing paired-end 100 -bp reads.

FastQC (https://www.bioinformatics.babraham.ac.uk/projects/fastqc/), was used to analyse raw read quality, and based on this information, the first $11 \mathrm{bp}$ of each read and any adapter sequences were removed using Trimmomatic 
(http://www.usadellab.org/cms/?page=trimmomatic). Bases were trimmed from reads using Sickle with a Phred quality threshold of 25 (https://github.com/najoshi/sickle). The resulting reads were mapped against the $P$. berghei ANKA genome (v36) using HISAT2 (version 2-2.1.0), using default parameters. Uniquely mapped, properly paired reads with mapping quality 40 or higher were retained using SAMtools (http://samtools.sourceforge.net/). Genome browser tracks were generated and viewed using the Integrative Genomic Viewer (IGV) (Broad Institute). Raw read counts were determined for each gene in the $P$. berghei genome using BedTools (https://bedtools.readthedocs.io/en/latest/\#) to intersect the aligned reads with the genome annotation. Differential expression analysis was done by use of $R$ package DESeq2 to call up- and down-regulated genes with an adjusted P-value cutoff of 0.05 . Gene ontology enrichment was done using $R$ package topGO (https://bioconductor.org/packages/release/bioc/html/topGO.html) with the weight01 algorithm.

\section{ChIP-seq analysis}

Gametocytes for kinesin-5GFP and kinesin-8XGFP parasites were harvested and the pellets were resuspended in $500 \mu \mathrm{l}$ of Hi-C lysis buffer $(25 \mathrm{mM}$ Tris- $\mathrm{HCl}, \mathrm{pH} 8.0,10 \mathrm{mM}$ $\mathrm{NaCl}, 2 \mathrm{mM} \mathrm{AESBF}, 1 \% \mathrm{NP}-40$, protease inhibitors). After incubation for $10 \mathrm{~min}$ at room temperature (RT), the resuspended pellets were homogenized by passing through a 26.5 gauge needle/syringe 15 times and cross-linked by adding formaldehyde (1.25\% final concentration) for $25 \mathrm{~min}$ at RT with continuous mixing. Crosslinking was stopped by adding glycine to a final concentration of $150 \mathrm{mM}$ and incubating for $15 \mathrm{~min}$ at RT with continuous mixing. The sample was centrifuged for $5 \mathrm{~min}$ at $2,500 \times \mathrm{g}(\sim 5,000 \mathrm{rpm})$ at $4^{\circ} \mathrm{C}$, the pellet washed once with $500 \mu \mathrm{l}$ ice-cold wash buffer $(50 \mathrm{mM}$ Tris- $\mathrm{HCl}, \mathrm{pH}$ 8.0, $50 \mathrm{mM} \mathrm{NaCl}, 1 \mathrm{mM}$ EDTA, $2 \mathrm{mM} \mathrm{AESBF}$, protease inhibitors) and the pellet stored at $80^{\circ} \mathrm{C}$ for ChIP seq analysis. The crosslinked parasite pellets were resuspended in $1 \mathrm{~mL}$ of nuclear extraction buffer (10 mM HEPES, $10 \mathrm{mM} \mathrm{KCl}, 0.1 \mathrm{mM}$ EDTA, $0.1 \mathrm{mM}$ EGTA, $1 \mathrm{mM}$ DTT, $0.5 \mathrm{mM}$ AEBSF, $1 \mathrm{X}$ protease inhibitor tablet), post $30 \mathrm{~min}$ incubation on ice, $0.25 \%$ Igepal-CA-630 was added and homogenized by passing through a $26 \mathrm{G} \times 1 / 2$ needle. The nuclear pellet extracted through $5000 \mathrm{rpm}$ centrifugation, was resuspended in $130 \mu \mathrm{l}$ of shearing buffer $(0.1 \%$ SDS, $1 \mathrm{mM}$ EDTA, $10 \mathrm{mM}$ Tris- $\mathrm{HCl} \mathrm{pH} 7.5,1 \mathrm{X}$ protease inhibitor tablet), and transferred to a $130 \mu \mathrm{l}$ Covaris sonication microtube. The sample was then sonicated using a Covaris S220 Ultrasonicator for 8 min (Duty cycle: $5 \%$, Intensity peak power: 140 , Cycles per burst: 200 , Bath temperature: $6^{\circ} \mathrm{C}$ ). The sample was transferred to ChIP dilution buffer (30 mM Tris-HCl pH 8, $3 \mathrm{mM}$ EDTA, 0.1\% SDS, $30 \mathrm{mM} \mathrm{NaCl}, 1.8 \%$ Triton X-100, $1 \mathrm{X}$ protease inhibitor tablet, $1 \mathrm{X}$ phosphatase inhibitor tablet) and centrifuged for $10 \mathrm{~min}$ at $13,000 \mathrm{rpm}$ at $4^{\circ} \mathrm{C}$, retaining the supernatant. For each sample, $13 \mu \mathrm{l}$ of protein A agarose/salmon sperm DNA beads were washed three times with $500 \mu \mathrm{l}$ ChIP dilution buffer (without inhibitors) by centrifuging for $1 \mathrm{~min}$ at $1000 \mathrm{rpm}$ at room temperature, then buffer was removed. For pre-clearing, the diluted chromatin samples were added to the beads and incubated for 1 hour at $4^{\circ} \mathrm{C}$ with rotation, then pelleted by centrifugation for $1 \mathrm{~min}$ at $1000 \mathrm{rpm}$. Supernatant was removed into a LoBind tube carefully so as not to remove any beads and $2 \mu \mathrm{g}$ of anti-GFP antibody (Abcam ab290, anti-rabbit) were added to the sample and incubated overnight at $4^{\circ} \mathrm{C}$ with rotation. Per sample, $25 \mu$ of protein A agarose/salmon sperm DNA beads were washed with ChIP dilution buffer (no inhibitors), blocked with 1 $\mathrm{mg} / \mathrm{mL}$ BSA for 1 hour at $4^{\circ} \mathrm{C}$, then washed three more times with buffer. $25 \mu \mathrm{l}$ of washed and blocked beads were added to the sample and incubated for 1 hour at $4^{\circ} \mathrm{C}$ with continuous mixing to collect the antibody/protein complex. Beads were pelleted by 
centrifugation for $1 \mathrm{~min}$ at $1000 \mathrm{rpm}$ at $4^{\circ} \mathrm{C}$. The bead/antibody/protein complex was then washed with rotation using $1 \mathrm{~mL}$ of each buffers twice; low salt immune complex wash buffer (1\% SDS, 1\% Triton X-100, 2 mM EDTA, $20 \mathrm{mM}$ Tris- $\mathrm{HCl}$ pH 8, $150 \mathrm{mM} \mathrm{NaCl}$ ), high salt immune complex wash buffer (1\% SDS, 1\% Triton X-100, 2 mM EDTA, 20 mM Tris- $\mathrm{HCl} \mathrm{pH} 8,500 \mathrm{mM} \mathrm{NaCl})$, high salt immune complex wash buffer $(1 \% \mathrm{SDS}, 1 \%$ Triton X-100, 2 mM EDTA, $20 \mathrm{mM}$ Tris-HCl pH 8, $500 \mathrm{mM} \mathrm{NaCl}$ ), TE wash buffer (10 $\mathrm{mM}$ Tris- $\mathrm{HCl} \mathrm{pH} 8,1 \mathrm{mM}$ EDTA) and eluted from antibody by adding $250 \mu \mathrm{l}$ of freshly prepared elution buffer ( $1 \%$ SDS, $0.1 \mathrm{M}$ sodium bicarbonate). We added $5 \mathrm{M} \mathrm{NaCl}$ to the elution and cross-linking was reversed by heating at $45^{\circ} \mathrm{C}$ overnight followed by addition of $15 \mu \mathrm{l}$ of $20 \mathrm{mg} / \mathrm{mL}$ RNAase A with $30 \mathrm{~min}$ incubation at $37^{\circ} \mathrm{C}$. After this, $10 \mu \mathrm{l} 0.5 \mathrm{M}$ EDTA, $20 \mu \mathrm{l} 1 \mathrm{M}$ Tris- $\mathrm{HCl} \mathrm{pH} 7.5$, and $2 \mu \mathrm{l} 20 \mathrm{mg} / \mathrm{mL}$ proteinase $\mathrm{K}$ were added to the elution and incubated for 2 hours at $45^{\circ} \mathrm{C}$. DNA was recovered by phenol/chloroform extraction and ethanol precipitation, using a phenol/chloroform/isoamyl alcohol (25:24:1) mixture twice and chloroform once, then adding $1 / 10$ volume of $3 \mathrm{M}$ sodium acetate $\mathrm{pH}$ $5.2,2$ volumes of $100 \%$ ethanol, and $1 / 1000$ volume of $20 \mathrm{mg} / \mathrm{mL}$ glycogen. Precipitation was allowed to occur overnight at $-20^{\circ} \mathrm{C}$. Samples were centrifuged at $13,000 \mathrm{rpm}$ for 30 $\min$ at $4^{\circ} \mathrm{C}$, then washed with fresh $80 \%$ ethanol, and centrifuged again for 15 min with the same settings. Pellet was air-dried and resuspended in $50 \mu l$ nuclease-free water. DNA was purified using Agencourt AMPure XP beads. Libraries were then prepared from this DNA using a KAPA library preparation kit (KK8230), and sequenced on a NovaSeq $6000 \quad$ machine. FastQC (https://www.bioinformatics.babraham.ac.uk/projects/fastqc/), was used to analyze raw read quality. Any adapter sequences were removed using Trimmomatic (http://www.usadellab.org/cms/?page=trimmomatic). Bases with Phred quality scores below 25 were trimmed using Sickle (https://github.com/najoshi/sickle). The resulting reads were mapped against the $P$. berghei ANKA genome (v36) using Bowtie2 (version 2.3.4.1). Using Samtools, only properly paired reads with mapping quality 40 or higher were retained, and reads marked as PCR duplicates were removed by PicardTools MarkDuplicates (Broad Institute). Genome browser tracks were generated and viewed using the Integrative Genomic Viewer (IGV).

\section{Statistical analysis}

All statistical analyses were performed using GraphPad Prism 7 (GraphPad Software). For qRT-PCR, an unpaired t-test was used to examine significant differences between wild-type and mutant strains.

\section{Acknowledgments}

We wish to thank Julie Rodgers for helping to maintain the insectary and other technical works.

Funding: This work was supported by: MRC UK (G0900109, G0900278, $\mathrm{MR} / \mathrm{K} 011782 / 1)$ and BBSRC (BB/N017609/1) to RT; the BBSRC (BB/N017609/1) to $\mathrm{MZ}$; the Francis Crick Institute, the Cancer Research UK (FC001097), the UK Medical Research Council (FC001097), and the Wellcome Trust (FC001097) to AAH; the NIH/NIAID (R01 Al136511) and the University of California, Riverside (NIFA-Hatch-225935) to KGLR; the Swiss National Science Foundation project grant (31003A_179321) to MB and BBSRC (BB/N018176/1) to CAM. This research was funded in whole, or in part, by the Wellcome Trust [FC001097]. For 
the purpose of Open Access, the author has applied a CC BY public copyright licence to any Author Accepted Manuscript version arising from this submission.

\section{Author contributions:}

Conceptualization: RT

Methodology: MZ, DB, RT, RR, SA, ZC, DJPF

Investigation: MZ, DB, RT, RR, SA, ZC, DJPF

Visualization: MZ, RT, RR, SA, DJPF

Supervision: RT, AAH, KGLR, CAM, MB

Writing一original draft: RT, MZ

Writing—review \& editing: RT, MZ, AAH, KGLR, CAM, MB, DJPF

Competing interests: All other authors declare they have no competing interests.

Data and materials availability: Sequence reads for ChIP-seq and RNA-seq have been deposited in the NCBI Sequence Read Archive with accession number: PRJNA731497. All other data are available in the main text or the supplementary materials.

\section{References}

Ali, A., S.N. Veeranki, A. Chinchole, and S. Tyagi. 2017. MLL/WDR5 Complex Regulates Kif2A Localization to Ensure Chromosome Congression and Proper Spindle Assembly during Mitosis. Dev Cell. 41:605-622 e607.

Andreadaki, M., T. Pace, F. Grasso, I. Siden-Kiamos, S. Mochi, L. Picci, L. Bertuccini, M. Ponzi, and C. Curra. 2020. Plasmodium berghei Gamete Egress Protein is required for fertility of both genders. Microbiologyopen. 9:e1038.

Balestra, A.C., M. Zeeshan, E. Rea, C. Pasquarello, L. Brusini, T. Mourier, A.K. Subudhi, N. Klages, P. Arboit, R. Pandey, D. Brady, S. Vaughan, A.A. Holder, A. Pain, D.J. Ferguson, A. Hainard, R. Tewari, and M. Brochet. 2020. A divergent cyclin/cyclindependent kinase complex controls the atypical replication of a malaria parasite during gametogony and transmission. Elife. 9.

Beetsma, A.L., T.J. van de Wiel, R.W. Sauerwein, and W.M. Eling. 1998. Plasmodium berghei ANKA: purification of large numbers of infectious gametocytes. Exp Parasitol. 88:69-72.

Bertiaux, E., A.C. Balestra, L. Bournonville, V. Louvel, B. Maco, D. Soldati-Favre, M. Brochet, P. Guichard, and V. Hamel. 2021. Expansion microscopy provides new insights into the cytoskeleton of malaria parasites including the conservation of a conoid. PLoS Biol. 19:e3001020.

Billker, O., V. Lindo, M. Panico, A.E. Etienne, T. Paxton, A. Dell, M. Rogers, R.E. Sinden, and H.R. Morris. 1998. Identification of xanthurenic acid as the putative inducer of malaria development in the mosquito. Nature. 392:289-292.

Briggs, L.J., J.A. Davidge, B. Wickstead, M.L. Ginger, and K. Gull. 2004. More than one way to build a flagellum: comparative genomics of parasitic protozoa. Curr Biol. 14:R611-612.

Bushell, E., A.R. Gomes, T. Sanderson, B. Anar, G. Girling, C. Herd, T. Metcalf, K. Modrzynska, F. Schwach, R.E. Martin, M.W. Mather, G.I. McFadden, L. Parts, G.G. Rutledge, A.B. Vaidya, K. Wengelnik, J.C. Rayner, and O. Billker. 2017. Functional Profiling of a Plasmodium Genome Reveals an Abundance of Essential Genes. Cell. 170:260-272 e268. 
Chan, K.Y., and K. Ersfeld. 2010. The role of the Kinesin-13 family protein TbKif13-2 in flagellar length control of Trypanosoma brucei. Mol Biochem Parasitol. 174:137140.

Dawson, S.C., M.S. Sagolla, J.J. Mancuso, D.J. Woessner, S.A. House, L. Fritz-Laylin, and W.Z. Cande. 2007. Kinesin-13 regulates flagellar, interphase, and mitotic microtubule dynamics in Giardia intestinalis. Eukaryot Cell. 6:2354-2364.

Depoix, D., S.R. Marques, D.J. Ferguson, S. Chaouch, T. Duguet, R.E. Sinden, P. Grellier, and L. Kohl. 2020. Vital role for Plasmodium berghei Kinesin8B in axoneme assembly during male gamete formation and mosquito transmission. Cell Microbiol. 22:e13121.

Ems-McClung, S.C., and C.E. Walczak. 2010. Kinesin-13s in mitosis: Key players in the spatial and temporal organization of spindle microtubules. Semin Cell Dev Biol. 21:276-282.

Fang, H., N. Klages, B. Baechler, E. Hillner, L. Yu, M. Pardo, J. Choudhary, and M. Brochet. 2017. Multiple short windows of calcium-dependent protein kinase 4 activity coordinate distinct cell cycle events during Plasmodium gametogenesis. Elife. 6.

Ferguson, D.J., F.L. Henriquez, M.J. Kirisits, S.P. Muench, S.T. Prigge, D.W. Rice, C.W. Roberts, and R.L. McLeod. 2005. Maternal inheritance and stage-specific variation of the apicoplast in Toxoplasma gondii during development in the intermediate and definitive host. Eukaryot Cell. 4:814-826.

Gambarotto, D., V. Hamel, and P. Guichard. 2021. Ultrastructure expansion microscopy (U-ExM). Methods Cell Biol. 161:57-81.

Gao, H., Z. Yang, X. Wang, P. Qian, R. Hong, X. Chen, X.Z. Su, H. Cui, and J. Yuan. 2018. ISP1-Anchored Polarization of GCbeta/CDC50A Complex Initiates Malaria Ookinete Gliding Motility. Curr Biol. 28:2763-2776 e2766.

Gould, S.B., W.H. Tham, A.F. Cowman, G.I. McFadden, and R.F. Waller. 2008. Alveolins, a new family of cortical proteins that define the protist infrakingdom Alveolata. Mol Biol Evol. 25:1219-1230.

Graumans, W., E. Jacobs, T. Bousema, and P. Sinnis. 2020. When Is a PlasmodiumInfected Mosquito an Infectious Mosquito? Trends Parasitol. 36:705-716.

Gubbels, M.J., C.D. Keroack, S. Dangoudoubiyam, H.L. Worliczek, A.S. Paul, C. Bauwens, B. Elsworth, K. Engelberg, D.K. Howe, I. Coppens, and M.T. Duraisingh. 2020. Fussing About Fission: Defining Variety Among Mainstream and Exotic Apicomplexan Cell Division Modes. Front Cell Infect Microbiol. 10:269.

Guttery, D.S., B. Poulin, D.J. Ferguson, B. Szoor, B. Wickstead, P.L. Carroll, C. Ramakrishnan, D. Brady, E.M. Patzewitz, U. Straschil, L. Solyakov, J.L. Green, R.E. Sinden, A.B. Tobin, A.A. Holder, and R. Tewari. 2012. A unique protein phosphatase with kelch-like domains (PPKL) in Plasmodium modulates ookinete differentiation, motility and invasion. PLoS Pathog. 8:e1002948.

Hammarton, T.C. 2019. Who Needs a Contractile Actomyosin Ring? The Plethora of Alternative Ways to Divide a Protozoan Parasite. Front Cell Infect Microbiol. 9:397.

Hirokawa, N., and Y. Tanaka. 2015. Kinesin superfamily proteins (KIFs): Various functions and their relevance for important phenomena in life and diseases. Exp Cell Res. 334:16-25.

Invergo, B.M., M. Brochet, L. Yu, J. Choudhary, P. Beltrao, and O. Billker. 2017. Subminute Phosphoregulation of Cell Cycle Systems during Plasmodium Gamete Formation. Cell Rep. 21:2017-2029. 
Iwanaga, S., T. Kato, I. Kaneko, and M. Yuda. 2012. Centromere plasmid: a new genetic tool for the study of Plasmodium falciparum. PLoS One. 7:e33326.

Janse, C.J., B. Franke-Fayard, G.R. Mair, J. Ramesar, C. Thiel, S. Engelmann, K. Matuschewski, G.J. van Gemert, R.W. Sauerwein, and A.P. Waters. 2006. High efficiency transfection of Plasmodium berghei facilitates novel selection procedures. Molecular and biochemical parasitology. 145:60-70.

Janse, C.J., B. Mons, R.J. Rouwenhorst, P.F. Van der Klooster, J.P. Overdulve, and H.J. Van der Kaay. 1985. In vitro formation of ookinetes and functional maturity of Plasmodium berghei gametocytes. Parasitology. 91 ( Pt 1):19-29.

Konjikusic, M.J., R.S. Gray, and J.B. Wallingford. 2021. The developmental biology of kinesins. Dev Biol. 469:26-36.

Kono, M., D. Prusty, J. Parkinson, and T.W. Gilberger. 2013. The apicomplexan inner membrane complex. Front Biosci (Landmark Ed). 18:982-992.

Koreny, L., M. Zeeshan, K. Barylyuk, E.C. Tromer, J.J.E. van Hooff, D. Brady, H. Ke, S. Chelaghma, D.J.P. Ferguson, L. Eme, R. Tewari, and R.F. Waller. 2021. Molecular characterization of the conoid complex in Toxoplasma reveals its conservation in all apicomplexans, including Plasmodium species. PLoS Biol. 19:e3001081.

Mair, G.R., J.A. Braks, L.S. Garver, J.C. Wiegant, N. Hall, R.W. Dirks, S.M. Khan, G. Dimopoulos, C.J. Janse, and A.P. Waters. 2006. Regulation of sexual development of Plasmodium by translational repression. Science. 313:667-669.

Mangione, M.C., and K.L. Gould. 2019. Molecular form and function of the cytokinetic ring. J Cell Sci. 132.

Manning, A.L., N.J. Ganem, S.F. Bakhoum, M. Wagenbach, L. Wordeman, and D.A. Compton. 2007. The kinesin-13 proteins Kif2a, Kif2b, and Kif2c/MCAK have distinct roles during mitosis in human cells. Mol Biol Cell. 18:2970-2979.

Marques, S.R., C. Ramakrishnan, R. Carzaniga, A.M. Blagborough, M.J. Delves, A.M. Talman, and R.E. Sinden. 2015. An essential role of the basal body protein SAS-6 in Plasmodium male gamete development and malaria transmission. Cell Microbiol. 17:191-206.

Mirvis, M., T. Stearns, and W. James Nelson. 2018. Cilium structure, assembly, and disassembly regulated by the cytoskeleton. Biochem J. 475:2329-2353.

Moon, R.W., C.J. Taylor, C. Bex, R. Schepers, D. Goulding, C.J. Janse, A.P. Waters, D.A. Baker, and O. Billker. 2009. A cyclic GMP signalling module that regulates gliding motility in a malaria parasite. PLoS Pathog. 5:e1000599.

Moores, C.A., M. Yu, J. Guo, C. Beraud, R. Sakowicz, and R.A. Milligan. 2002. A mechanism for microtubule depolymerization by Kinl kinesins. Mol Cell. 9:903909.

Morano, A.A., and J.D. Dvorin. 2021. The Ringleaders: Understanding the Apicomplexan Basal Complex Through Comparison to Established Contractile Ring Systems. Front Cell Infect Microbiol. 11:656976.

Morrissette, N.S., and L.D. Sibley. 2002. Cytoskeleton of apicomplexan parasites. Microbiol Mol Biol Rev. 66:21-38; table of contents.

Okamoto, N., T.P. Spurck, C.D. Goodman, and G.I. McFadden. 2009. Apicoplast and mitochondrion in gametocytogenesis of Plasmodium falciparum. Eukaryot Cell. 8:128-132.

Pandey, R., S. Abel, M. Boucher, R.J. Wall, M. Zeeshan, E. Rea, A. Freville, X.M. Lu, D. Brady, E. Daniel, R.R. Stanway, S. Wheatley, G. Batugedara, T. Hollin, A.R. Bottrill, D. Gupta, A.A. Holder, K.G. Le Roch, and R. Tewari. 2020. Plasmodium Condensin Core Subunits SMC2/SMC4 Mediate Atypical Mitosis and Are 
Essential for Parasite Proliferation and Transmission. Cell Rep. 30:1883-1897 e1886.

Pfander, C., B. Anar, M. Brochet, J.C. Rayner, and O. Billker. 2013. Recombinationmediated genetic engineering of Plasmodium berghei DNA. Methods Mol Biol. 923:127-138.

Pfander, C., B. Anar, F. Schwach, T.D. Otto, M. Brochet, K. Volkmann, M.A. Quail, A. Pain, B. Rosen, W. Skarnes, J.C. Rayner, and O. Billker. 2011. A scalable pipeline for highly effective genetic modification of a malaria parasite. Nat Methods. 8:1078-1082.

Philip, N., and A.P. Waters. 2015. Conditional Degradation of Plasmodium Calcineurin Reveals Functions in Parasite Colonization of both Host and Vector. Cell Host Microbe. 18:122-131.

Piao, T., M. Luo, L. Wang, Y. Guo, D. Li, P. Li, W.J. Snell, and J. Pan. 2009. A microtubule depolymerizing kinesin functions during both flagellar disassembly and flagellar assembly in Chlamydomonas. Proc Natl Acad Sci U S A. 106:47134718.

Reid, A.J., A.M. Talman, H.M. Bennett, A.R. Gomes, M.J. Sanders, C.J.R. Illingworth, O. Billker, M. Berriman, and M.K. Lawniczak. 2018. Single-cell RNA-seq reveals hidden transcriptional variation in malaria parasites. Elife. 7.

Schrevel, J., G. Asfaux-Foucher, and J.M. Bafort. 1977. [Ultrastructural study of multiple mitoses during sporogony of Plasmodium b. berghei]. J Ultrastruct Res. 59:332350.

Sebastian, S., M. Brochet, M.O. Collins, F. Schwach, M.L. Jones, D. Goulding, J.C. Rayner, J.S. Choudhary, and O. Billker. 2012. A Plasmodium calcium-dependent protein kinase controls zygote development and transmission by translationally activating repressed mRNAs. Cell Host Microbe. 12:9-19.

Sehring, I.M., B. Dong, E. Denker, P. Bhattachan, W. Deng, B.T. Mathiesen, and D. Jiang. 2014. An equatorial contractile mechanism drives cell elongation but not cell division. PLoS Biol. 12:e1001781.

Sinden, R.E. 1991. Mitosis and meiosis in malarial parasites. Acta Leiden. 60:19-27.

Sinden, R.E., E.U. Canning, R.S. Bray, and M.E. Smalley. 1978. Gametocyte and gamete development in Plasmodium falciparum. Proc $R$ Soc Lond B Biol Sci. 201:375-399.

Sinden, R.E., and K. Strong. 1978. An ultrastructural study of the sporogonic development of Plasmodium falciparum in Anopheles gambiae. Trans $R$ Soc Trop Med Hyg. 72:477-491.

Sinden, R.E., A. Talman, S.R. Marques, M.N. Wass, and M.J. Sternberg. 2010. The flagellum in malarial parasites. Curr Opin Microbiol. 13:491-500.

Spreng, B., H. Fleckenstein, P. Kubler, C. Di Biagio, M. Benz, P. Patra, U.S. Schwarz, M. Cyrklaff, and F. Frischknecht. 2019. Microtubule number and length determine cellular shape and function in Plasmodium. EMBO J. 38:e100984.

Straschil, U., A.M. Talman, D.J. Ferguson, K.A. Bunting, Z. Xu, E. Bailes, R.E. Sinden, A.A. Holder, E.F. Smith, J.C. Coates, and T. Rita. 2010. The Armadillo repeat protein PF16 is essential for flagellar structure and function in Plasmodium male gametes. PLoS One. 5:e12901.

Talman, A.M., J.H. Prieto, S. Marques, C. Ubaida-Mohien, M. Lawniczak, M.N. Wass, T. Xu, R. Frank, A. Ecker, R.S. Stanway, S. Krishna, M.J. Sternberg, G.K. Christophides, D.R. Graham, R.R. Dinglasan, J.R. Yates, 3rd, and R.E. Sinden. 2014. Proteomic analysis of the Plasmodium male gamete reveals the key role for glycolysis in flagellar motility. Malar J. 13:315. 
Tewari, R., D. Dorin, R. Moon, C. Doerig, and O. Billker. 2005. An atypical mitogenactivated protein kinase controls cytokinesis and flagellar motility during male gamete formation in a malaria parasite. Mol Microbiol. 58:1253-1263.

Tewari, R., U. Straschil, A. Bateman, U. Bohme, I. Cherevach, P. Gong, A. Pain, and O. Billker. 2010. The systematic functional analysis of Plasmodium protein kinases identifies essential regulators of mosquito transmission. Cell Host Microbe. 8:377387.

Tremp, A.Z., E.I. Khater, and J.T. Dessens. 2008. IMC1b is a putative membrane skeleton protein involved in cell shape, mechanical strength, motility, and infectivity of malaria ookinetes. J Biol Chem. 283:27604-27611.

Vasudevan, K.K., Y.Y. Jiang, K.F. Lechtreck, Y. Kushida, L.M. Alford, W.S. Sale, T. Hennessey, and J. Gaertig. 2015. Kinesin-13 regulates the quantity and quality of tubulin inside cilia. Mol Biol Cell. 26:478-494.

Vavylonis, D., J.Q. Wu, S. Hao, B. O'Shaughnessy, and T.D. Pollard. 2008. Assembly mechanism of the contractile ring for cytokinesis by fission yeast. Science. 319:97-100.

Verhey, K.J., and J.W. Hammond. 2009. Traffic control: regulation of kinesin motors. Nat Rev Mol Cell Biol. 10:765-777.

Vicente, J.J., and L. Wordeman. 2015. Mitosis, microtubule dynamics and the evolution of kinesins. Exp Cell Res. 334:61-69.

Volkmann, K., C. Pfander, C. Burstroem, M. Ahras, D. Goulding, J.C. Rayner, F. Frischknecht, O. Billker, and M. Brochet. 2012. The alveolin IMC1h is required for normal ookinete and sporozoite motility behaviour and host colonisation in Plasmodium berghei. PLoS One. 7:e41409.

Wall, R.J., M. Zeeshan, N.J. Katris, R. Limenitakis, E. Rea, J. Stock, D. Brady, R.F. Waller, A.A. Holder, and R. Tewari. 2019. Systematic analysis of Plasmodium myosins reveals differential expression, localisation, and function in invasive and proliferative parasite stages. Cell Microbiol. 21:e13082.

Wang, X., P. Qian, H. Cui, L. Yao, and J. Yuan. 2020. A protein palmitoylation cascade regulates microtubule cytoskeleton integrity in Plasmodium. EMBO J. 39:e104168.

Wickstead, B., and K. Gull. 2006. A "holistic" kinesin phylogeny reveals new kinesin families and predicts protein functions. Mol Biol Cell. 17:1734-1743.

Wickstead, B., and K. Gull. 2011. The evolution of the cytoskeleton. J Cell Biol. 194:513525.

Wickstead, B., K. Gull, and T.A. Richards. 2010. Patterns of kinesin evolution reveal a complex ancestral eukaryote with a multifunctional cytoskeleton. BMC Evol Biol. 10:110.

Yount, A.L., H. Zong, and C.E. Walczak. 2015. Regulatory mechanisms that control mitotic kinesins. Exp Cell Res. 334:70-77.

Zeeshan, M., D. Brady, R.R. Stanway, C.A. Moores, A.A. Holder, and R. Tewari. 2020a. Plasmodium berghei Kinesin-5 Associates With the Spindle Apparatus During Cell Division and Is Important for Efficient Production of Infectious Sporozoites. Front Cell Infect Microbiol. 10:583812.

Zeeshan, M., D.J. Ferguson, S. Abel, A. Burrrell, E. Rea, D. Brady, E. Daniel, M. Delves, S. Vaughan, A.A. Holder, K.G. Le Roch, C.A. Moores, and R. Tewari. 2019a. Kinesin-8B controls basal body function and flagellum formation and is key to malaria transmission. Life Sci Alliance. 2.

Zeeshan, M., R. Pandey, D.J.P. Ferguson, E.C. Tromer, R. Markus, S. Abel, D. Brady, E. Daniel, R. Limenitakis, A.R. Bottrill, K.G. Le Roch, A.A. Holder, R.F. Waller, 


\section{Figure legends}

Fig 1. Kinesin pattern of expression and diverse subcellular locations at various stages in the Plasmodium berghei life cycle. (A) Life cycle of Plasmodium spp. Showing different proliferative and invasive stages in its host and vector. (B) A summary of expression and location of kinesins-GFP during different stages of $P$. berghei life cycle. (C) Live cell imaging showing subcellular locations of nine kinesin-GFP proteins (green) during various stages of the $P$. berghei life cycle. DNA is stained with Hoechst dye (blue). Arrowhead indicates basal end and arrow indicates apical end of the ookinete. $\mathrm{Mpa}=\min$ post activation. Oocyst-NS: non-sporulating oocyst, Oocyst-S: sporulating oocyst. Scale bar $=5 \mu \mathrm{m}$.

Fig 2. Nuclear kinesins (kinesin-5 and $-8 \mathrm{X}$ ) associate with kinetochore and bind to the centromere during male gamete formation. (A) Live cell imaging showing the temporal dynamics of kinesin-5GFP (green) along with kinetochore marker NDC80Cherry (red) during male gametogenesis. (B) Live cell imaging showing the dynamics of kinesin-8XGFP (green) along with kinetochore marker NDC80Cherry (red) during male gametogenesis. (C) ChIP-seq analysis of kinesin-5 and kinesin-8X during gametocyte stage. Lines on top are division points between chromosomes and circles on the bottom indicate locations of centromeres. DNA is stained with Hoechst dye (blue); $\mathrm{mpa}=\mathrm{min}$ post activation; Scale bar $=5 \mu \mathrm{m}$.

Fig 3. Apicomplexa-enriched kinesins (kinesin-X3 and $-X 4$ ) are located at the pellicle during ookinete differentiation and axonemes in male gametogenesis, respectively. (A) Live cell imaging showing temporal location of kinesin-X3 (green) associated with pellicle formation (arrows) during zygote to ookinete transition (2-24 h). A cy3-conjugated antibody, 13.1, which recognises the protein P28 on the surface of zygote and ookinete stages, was used to track these stages (red). DNA is stained with Hoechst dye (blue). Scale bar=5 $\mu \mathrm{m}$. (B) Live cell imaging shows the association of kinesin-X4 (green) with axoneme marker kinesin-8B (red) during male gametogenesis. Note in later stages, axonemes (arrows) are labelled with both markers. DNA is stained with Hoechst dye (blue). $\mathrm{mpa}=\min$ post activation. Scale bar $=5 \mu \mathrm{m}$.

Fig 4. Phenotypic screen of nine kinesins in Plasmodium reveals their role during sexual and transmission stages within mosquito vector. (A) Summary of phenotypes at various stages of life cycle resulting from gene deletion. The phenotype 
301 was examined for asexual blood stage development (schizogony), exflagellation (male gamete formation), ookinete formation, oocyst number, oocyst size, sporozoite formation in oocyst, presence of salivary gland sporozoites and sporozoite transmission to vertebrate host. $N / D=N o t$ Determined (B) Male gametogenesis in kinesin gene-deletion lines in comparison with WTGFP parasite, measured as the number of exflagellation centres per field; more than 20 fields were counted for each line. Mean \pm SEM. $n=3$ independent experiments. (C) Percentage ookinete conversion comparing knockout and WTGFP parasites. Ookinetes were identified using 13.1 antibody for surface marker (P28, red) and defined as those cells that differentiated successfully into elongated 'banana shaped' ookinetes. Mean \pm SEM. $\mathrm{n}=5$ independent experiments. (D) Total number of GFP-positive oocysts per mosquito midgut at 7-, 14- and 21-days post infection for knockout and WTGFP parasites; at least 10 mosquito midguts were counted for each line. Mean \pm SEM. $n=3$ independent experiments. (E) Representative mosquito midguts at 10x and 63x magnification showing oocysts of kinesin knockout and WTGFP lines at 7-, 14- and 21-days post infection. Scale bar $=50 \mu \mathrm{M}(10 \mathrm{x}), 20 \mu \mathrm{M}$ (63x). (F) Total number of sporozoites in oocysts of kinesin knockout and WTGFP parasites at 14and 21-days post infection. Mean \pm SEM. $n=3$ independent experiments. (G) Total number of sporozoites in salivary glands of kinesin knockout and WT-GFP parasites. Mean \pm SEM. $\mathrm{n}=3$ independent experiments. $(\mathrm{H})$ Mosquito bite back experiments showing no transmission of $\Delta k$ kinesin- $8 B$ and $\Delta$ kinesin- $8 X$ parasites, while other kinesin knockout and WTGFP parasites show successful transmission from mosquito to mice. Mean \pm SEM. $\mathrm{n}=3$ independent experiments. ${ }^{*} p \leq 0.05,{ }^{* *} p \leq 0.01$ and ${ }^{* * *} p \leq 0.001$.

Fig 5. Kinesin-20 makes a ring like structure at the base of developing ookinete and is important for zygote to ookinete transformation and subsequent motility. (A) Live cell imaging showing localization of kinesin-20GFP (green) in the female gametocyte, zygote and during ookinete transformation. Kinesin-20GFP accumulates at the neck of developing ookinete making a ring like structure (indicated by arrows). Labelling with P28 was used to identify the surface of activated female gametes, zygotes and ookinetes and to mark these stages (red). DNA is labelled with Hoechst dye (blue) (B). Ookinete developmental stages for $\Delta$ kinesin-20 and WTGFP parasites. Ookinetes were identified using P28 and defined as those cells differentiated successfully into elongated 'banana shaped' forms. Round cells are arrested zygotes or female gametocytes, while bulbous cells did not develop to banana-shaped ookinetes for $\Delta$ kinesin-20 parasites. DNA is stained with Hoechst dye (blue). Scale bar $=5 \mu \mathrm{m}$. (C) Ookinete conversion as a percentage of cells for $\Delta$ kinesin-20 and WTGFP parasites after $24 \mathrm{~h}$. Mean \pm SEM. $\mathrm{n}=4$ independent experiments. (D) Representative frames from time-lapse videos of motile WTGFP ookinetes and sessile ookinetes of $\Delta$ kinesin-20 in Matrigel. Black arrow indicates apical end of ookinete. Scale bar $=5 \mu \mathrm{m}$. (E) Ookinete motility for WT-GFP and $\Delta$ kinesin-20 lines in Matrigel. More than 20 ookinetes were examined for each line. Mean \pm SEM. $n=3$ independent experiments. (F) Representative frames from time-lapse videos showing motile sporozoites of WTGFP and $\Delta$ kinesin-20 lines. Black arrow indicates the apical end of the sporozoites. Scale bar $=5 \mu \mathrm{m}$. (G) Sporozoite motility for WTGFP and $\Delta$ kinesin-20 lines. More than 20 sporozoites were examined for each line. Mean \pm SEM. $n=3$ independent experiments. (H) qRT-PCR analysis of transcripts for other kinesin genes in $\triangle k$ kinesin-20 and WTGFP parasites. Mean \pm SEM. $\mathrm{n}=3$ independent experiments. $\mathrm{Ns}=$ not significant. (I) RNAseq analysis showing no transcript in $\Delta$ kinesin-20 parasites. (J) Volcano plot showing differentially regulated genes in $\Delta$ kinesin-20 gametocytes activated for $2 \mathrm{~h}$. 
351 Fig 6. Ultrastructural analysis of WTGFP and $\Delta$ kinesin-20 ookinetes. (A)

352 Representative confocal images of expanded ookinetes of WTGFP and bulbous cells of $\Delta$ kinesin-20 lines stained for $\alpha$ - and $\beta$-tubulin (magenta) showing sub-pellicle MTs. Scale bar $=1 \mu \mathrm{m}$ (B) Electron micrographs of WTGFP $(\mathbf{a}, \mathbf{d}, \mathbf{f}, \mathbf{h})$ and $\Delta$ kinesin-20 (b, c, e, g, i) ookinetes. Bars represent $1 \mu \mathrm{m}(\mathrm{a}-\mathrm{c})$ and $100 \mathrm{~nm}(\mathrm{~d}-\mathrm{i})$.

(a) Longitudinal section of a crescentic shaped WTGFP ookinete showing the apical complex (AC) with numerous micronemes $(\mathrm{M})$ in the anterior cytoplasm and a more posteriorly located crystalline body $(\mathrm{Cr})$ and nucleus $(\mathrm{N})$. (b) Longitudinal section through an immature $\Delta$ kinesin-20 ookinete displaying a bulbous shape. Within the cytoplasm, the apical complex (AC) plus an irregular shaped nucleus $(\mathrm{N})$ and a few irregularly arranged dense granules (DG) can be identified. (c). Longitudinal section through a more mature $\Delta$ kinesin-20 ookinete showing a bulbous shaped cell. Within the cytoplasm the apical complex (AC), nucleus (N) and crystalline body $(\mathrm{Cr})$ can be identifying but note the micronemes (M) appear to be distributed throughout the cytoplasm. (d, e) Details of longitudinal sections through the apical complex of WTGFP (d) and $\Delta$ kinesin-20 (e) ookinetes showing similar substructures consisting of three conoidal rings $(\mathrm{CR})$, an anterior polar ring 1 (P1) formed by the IMC and a second polar ring (P2) representing the initiation site for the sub-pellicular microtubules (Mt). The polar rings are separated by a collar consisting of an outer electron dense layer (cd) and an inner electron lucent layer (cl). Note the micronemes (M) with ducts (D) direct to the anterior plasmalemma. (f, g) Cross section through the periphery of the anterior complex of a WTGFP (f) and a $\Delta$ kinesin-20 (g) parasite showing similar sub-structure consisting of the outer plasmalemma (PM) and the underlying inner membrane complex (IMC) which appears fused to the outer electron dense (cd) region of the apical collar while the more electron lucent inner region is in close contact with sub-pellicular microtubules (Mt). (h, i) Cross section of the pellicle posterior to the apical complex consisting of the outer plasmalemma and the underlying IMC. However, note that while the sub-pellicular microtubules (Mt) in the WTGFP parasite (h) are evenly spaced, those of the $\Delta$ kinesin20 (i) show irregular spacing and some clumping.

Fig 7. Kinesin-13 associates with kinetochore marker, NDC80, during various proliferative stages of parasite life cycle. Live cell imaging showing location of kinesin-13GFP (green) with respect to kinetochore marker NDC80Cherry (red) during asexual (A, B) and sexual (C, D) proliferative stages. Asexual proliferative stages include endomitosis during blood schizogony and sporogony. Sexual stages include endomitosis during male gametogenesis and meiosis during ookinete development. Kinesin-13GFP (green) shows a diffuse distribution in the cytoplasm, together with strong foci at different stages of development that co-localise with NDC80Cherry (red). DNA is stained with Hoechst dye (blue). Scale bar $=5 \mu \mathrm{m}$.

389

390

391

392

393

394

395

396

397

398

399

400

Fig 8. Phenotypic analysis of gene expression knockdown in kinesin-13PTD transgenic line at various proliferative stages during the life cycle (A) qRTPCR analysis showing down-regulation of kinesin-13 gene in kinesin-13PTD parasites compared to WTGFP. Mean \pm SEM, $n=3$ independent experiments. (B) Exflagellation centres per field at 15 min post-activation. $n=3$ independent experiments $(>20$ fields per experiment). Error bar, \pm SEM. (C) Percentage ookinete conversion from zygote. $n=3$ independent experiments (> 80 cells). Error bar, \pm SEM. (D) Oocysts at days 7,14 , and 21 post infection. $\mathrm{n}=3$ independent experiments with a minimum of 8 mosquito guts. Error bar, \pm SEM. (E) Bite back experiments reveal no transmission of kinesin-13PTD and successful transmission of WTGFP parasites from mosquito to mouse. Mean \pm 
401 SEM. $\mathrm{n}=3$ independent experiments. (F) qRT-PCR analysis of transcripts for other

kinesin genes in kinesin-13PTD compared to WTGFP parasites. $n=3$ independent experiments. Error bar, \pm SEM. (G) RNAseq analysis showing depletion of kinesin-13 transcript in kinesin-13PTD parasites. (H) Volcano plot showing differentially regulated genes in kinesin-13PTD gametocytes before activation (0 min). (I) Volcano plot showing differentially regulated genes in kinesin-13PTD gametocytes activated for $15 \mathrm{~min}$. (J) Heat maps showing differential regulation of various genes involved in axoneme biogenesis, IMC/glideosome and chromosome dynamics. ${ }^{*} p \leq 0.05,{ }^{* *} p \leq 0.01$ and ${ }^{* * *} p \leq 0.001$.

Fig 9. Ultrastructural analysis of WTGFP and kinesin-13PTD gametocytes and ookinetes. (A) Representative confocal images of expanded gametocytes of WTGFP and kinesin-13PTD lines stained for $\alpha$ - and $\beta$-tubulin (magenta) showing labelling of spindle (arrow) and axonemal MTs (arrowhead) at 4-5 min and axonemal MTs (arrowhead) at $15 \mathrm{~min}$ post activation. Similarly, representative confocal images of expanded ookinetes of WTGFP and kinesin-13PTD lines stained for $\alpha$ - and $\beta$-tubulin (magenta) showing well-organised subpellicular MTs (white arrows) and apical tubulin ring (ATR, red arrows) in WTGFP ookinetes and disorganised MTs were observed in kinesin-13PTD. Scale bars $=1 \mu \mathrm{m}$. (B) Electron micrographs of $P$. berghei male gametogenesis of WTGFP $(\mathbf{a}, \mathbf{b}, \mathbf{e}, \mathbf{f}, \mathbf{g})$ and kinesin-13PTD $(\mathbf{c}, \mathbf{d}, \mathbf{h}, \mathbf{i})$ at 6 min $(\mathbf{a}-\mathbf{d})$ and 15 min $(\mathbf{e}-\mathbf{i})$ post-activation. Bars represent $1 \mu \mathrm{m}$ in $\mathbf{a}, \mathbf{c}, \mathbf{e}, \mathbf{h}$ and $100 \mathrm{~nm}$ in other micrographs. (a) Early stage WTGFP showing the large central nucleus (N) displaying two nuclear poles (NP) with cytoplasm containing a number of axonemes (A). (b) Detail of the cytoplasm illustrating a number of normal 9+2 axonemes $(A)$ and abnormal axonemes. (c) Early stage kinesin-13PTD gametocytes showing the central nucleus (N) but the cytoplasm lacked visible axonemes. (d) Detail of the enclosed area in c showing randomly arranged free single and duplet MTs with some forming partial axonemes. (e) Late stage WTGFP showing a flagellum (F) of a developing microgamete emerging from the male gametocyte (exflagellation). $\mathrm{N}$ - nucleus; Ch- chromatin. (f) Detail of a longitudinal section through a free male gamete showing the nucleus $(N)$ and flagellum $(F)$. (g) Detail of a cross section through a male gamete showing the 9+2 flagellum and the nucleus (arrowhead). (h) Late-stage kinesin-13PTD gametocyte showing the central nucleus $(\mathrm{N})$ with clumps of chromatin $(\mathrm{Ch})$ but an absence of any $9+2$ axonemes. (i) Detail of the enclosed area in $\mathbf{h}$ showing a microtubule (Mt) enclosed by the plasmalemma emerging from the male gametocyte.

Fig 10. Summary of kinesin function throughout the $\boldsymbol{P}$. berghei life cycle. Kinesins with important roles in host and mosquito are highlighted in red.

\section{Supplementary Materials}

\section{Supplementary figures}

\section{Fig S1. Generation and genotypic analysis of kinesin-GFP parasites}

A. Schematic representation of the endogenous kinesin locus, the GFP-tagging construct and the recombined kinesin locus following single homologous recombination. Arrows 1 (P1) and 3 (P3) indicate the position of PCR primers used to confirm successful integration of the construct. B. Diagnostic PCR of kinesin and WT parasites using primers: integration primer (P1) and ol492 (P2). The bands of expected size for amplified DNA fragments are indicated for each tag line. 
Fig S2. The location of kinesin- 5 and kinesin- $8 X$ in relation to that of the axoneme marker (kinesin-8B)

\begin{abstract}
A. The location of kinesin-5GFP (green) in relation to the axoneme marker, kinesin8BCherry (red) during male gamete formation. B. The location of kinesin-8XGFP (green) in relation to the axoneme marker, kinesin-8BCherry (red) during male gamete formation. The nuclear location of kinesin- 5 and kinesin-8X contrasts with the cytoplasmic location of kinesin-8B during chromosome replication and segregation, indicating that kinesin- 5 and kinesin- $8 \mathrm{X}$ are associated with the mitotic spindle. $\mathrm{mpa}=\min$ post activation. DNA is stained with Hoechst dye (blue). Scale bar $=5 \square \mu \mathrm{m}$.
\end{abstract}

\title{
Fig S3. Generation and genotypic analysis of $\Delta$ kinesin parasites
}

A. Schematic representation of the endogenous kinesin locus, the targeting knockout construct and the recombined kinesin locus following double homologous crossover recombination. Arrows 1 and 2 indicate PCR primers used to confirm successful integration in the kinesin locus following recombination and arrows 3 and 4 indicate PCR primers used to show deletion of the kinesin gene. B. Integration PCR of the kinesin locus in WTGFP (WT) and knockout (Mut) parasites using primers: integration primer and ol248. Integration of the targeting construct gives expected size band for each gene. C. qRT-PCR analysis of transcript in WT-GFP and $\Delta$ kinesin parasites confirming the deletion of respected genes.

\section{Fig S4. Quantification of MT length in $\Delta$ kinesin-20 and WTGFP ookinetes}

The MT lengths were measured from the images of ookinetes stained to reveal $\alpha$ and $\beta$ tubulins obtained by expansion microscopy. The bar diagram shows the length of subpellicular MTs in bulbous $\Delta$ kinesin-20 ookinetes compared to WTGFP ookinetes. Mean \pm SEM. $n=3$ independent experiments.

Fig S5. Kinesin-13 shows co-localisation with tubulins in male gametocyte and gamete

(A) Indirect immunofluorescence assay showing the co-localisation of kinesin-13 (green) and tubulin staining (red) in male gamete. Scale bar $=5 \square \mu \mathrm{m}$. (B) Expansion microscopy showing co-localization of kinesin-13 (green) with $\alpha / \beta$ tubulin staining (purple) in gametocytes activated for $15 \mathrm{~min} . \mathrm{mpa}=\min$ post activation. Scale bar $=$ $1 \square \mu \mathrm{m}$.

Fig S6. Generation and genotype analysis of conditional knockdown kinesin13 parasites

(A) Schematic representation of auxin inducible degron (AID) strategy to generate kinesin-13AID/HA parasites. (B) Integration PCR of the kinesin-13AID/HA construct in the kinesin-13 locus. Oligonucleotides used for PCR genotyping are indicated and agarose gels for corresponding PCR products from genotyping reactions are shown. (C) Kinesin-13AID/HA protein expression level as measured by western blotting upon addition of auxin to mature purified gametocytes; a-tubulin serves as a loading control. (D) Schematic representation of the promoter swap strategy (kinesin-13PTD, placing kinesin-13 under the control of the clag promoter) by double homologous recombination. 
499 Arrows 1 and 2 indicate the primer positions used to confirm 5 integration and arrows 3

500 and 4 indicate the primers used for 3 ' integration. (E) Integration PCR of the promotor 501 swap construct into the kinesin-13 locus. Primer 1 (5'-IntPTD50) with primer 2 (5'502 IntPTD) were used to determine successful integration of the selectable marker. Primer 3 503 (3'-intClag) and primer 4 (3'-IntPTD50) were used to determine the successful integration 504 of the clag promoter. Primer 1 (5'-IntPTD50) and primer 4 (3'-IntPTD50) were used to 505 show complete knock-in of the construct and the absence of a band at $2.3 \mathrm{~kb}$ 506 (endogenous) expected if no integration occurred.

\section{Supplementary Tables}

509 Table S1. List of genes differentially expressed between $\triangle$ kinesin-20 and WTGFP

510 activated gametocytes for $2 \mathrm{~h}$ (table S1A); and between kinesin-13PTD and WTGFP

511 gametocytes (table S1B and S1C)

Table S2. Oligonucleotides used in this study.

\section{Supplementary Movies}

Movie S1: Gliding motility of WT-GFP ookinetes

Movie S2: Gliding motility of $\Delta$ kinesin-20 ookinetes

Movie S3: Gliding motility of WT-GFP salivary gland sporozoite

Movie S4: Gliding motility $\Delta k i n e s i n-20$ salivary gland sporozoite 
A
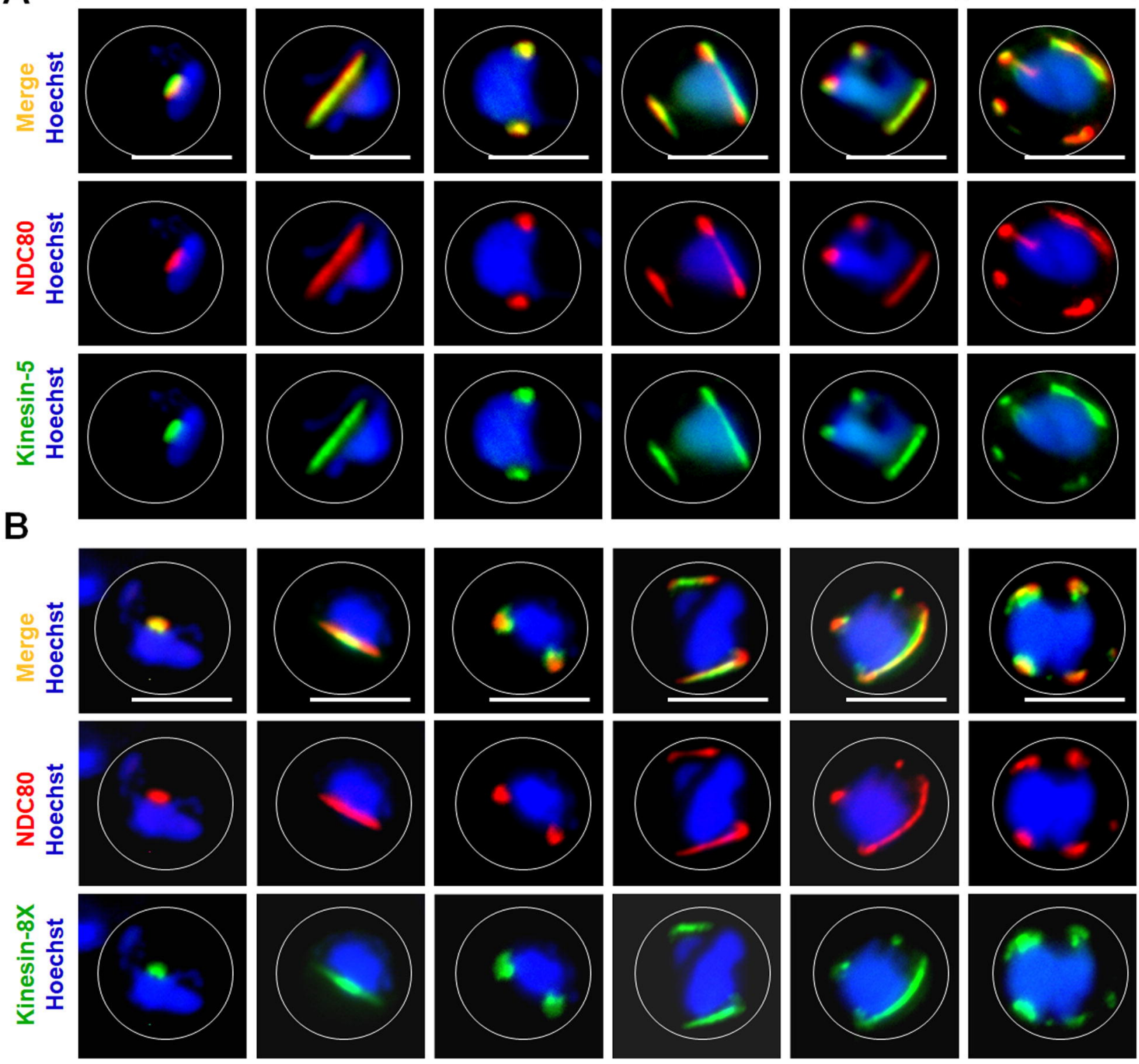

C

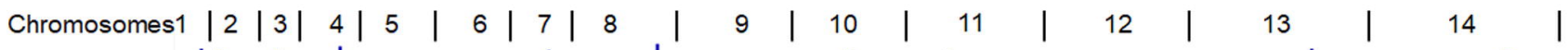
kinesin-5

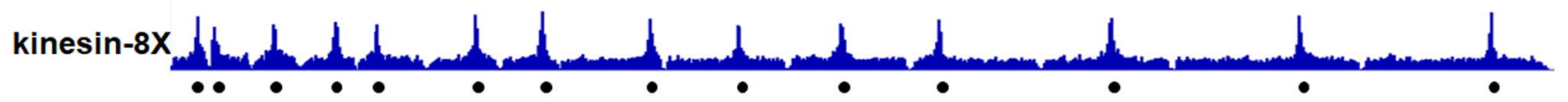




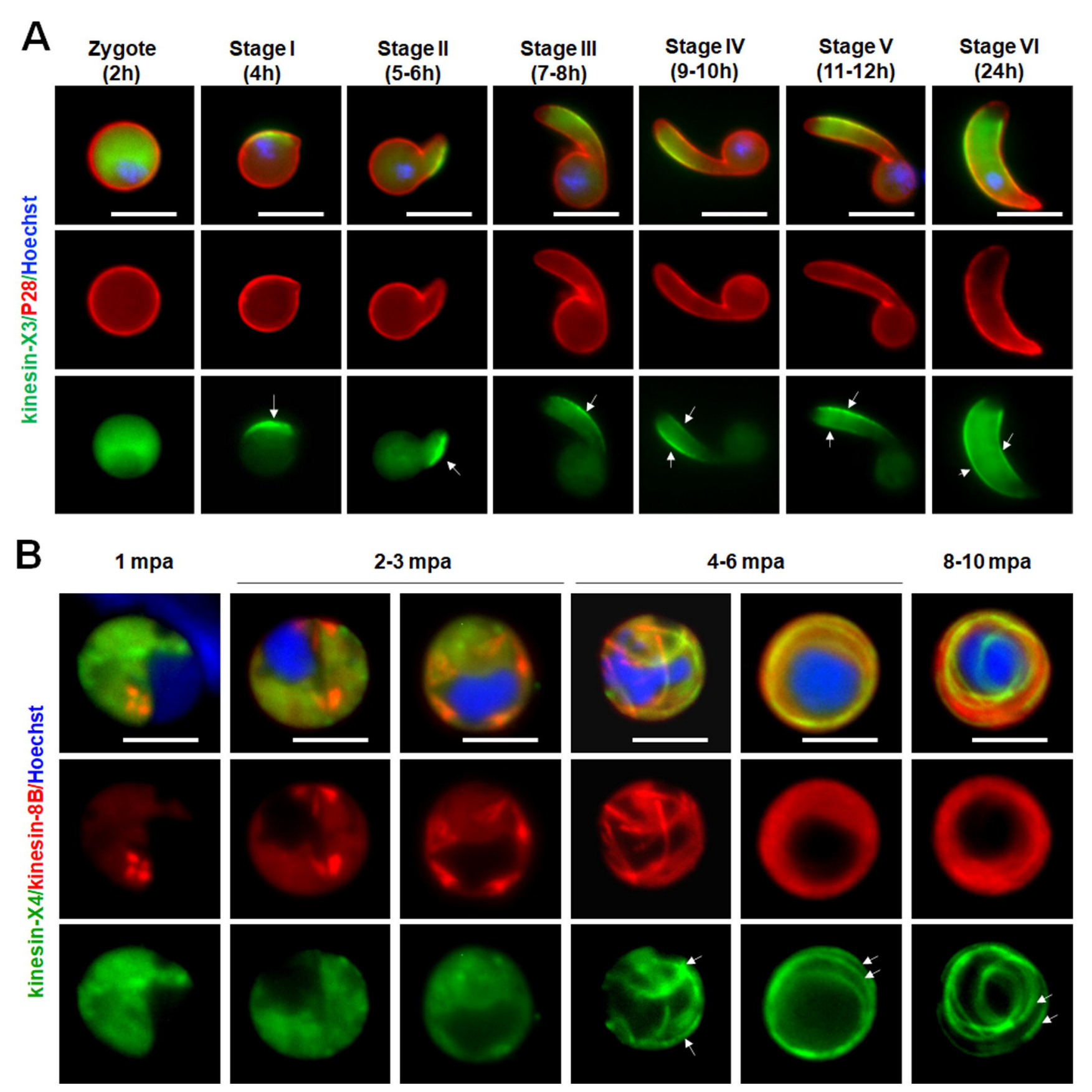




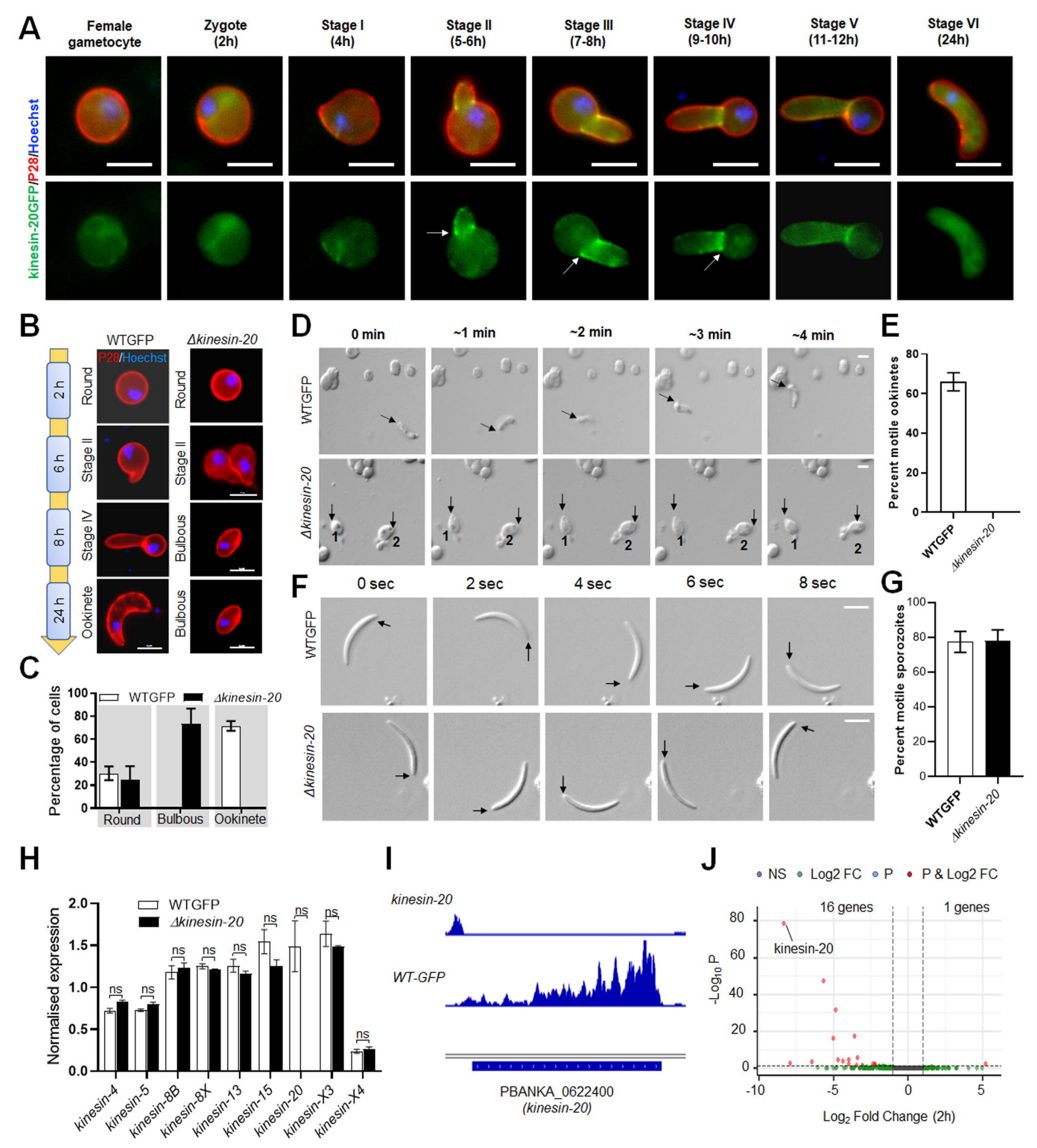



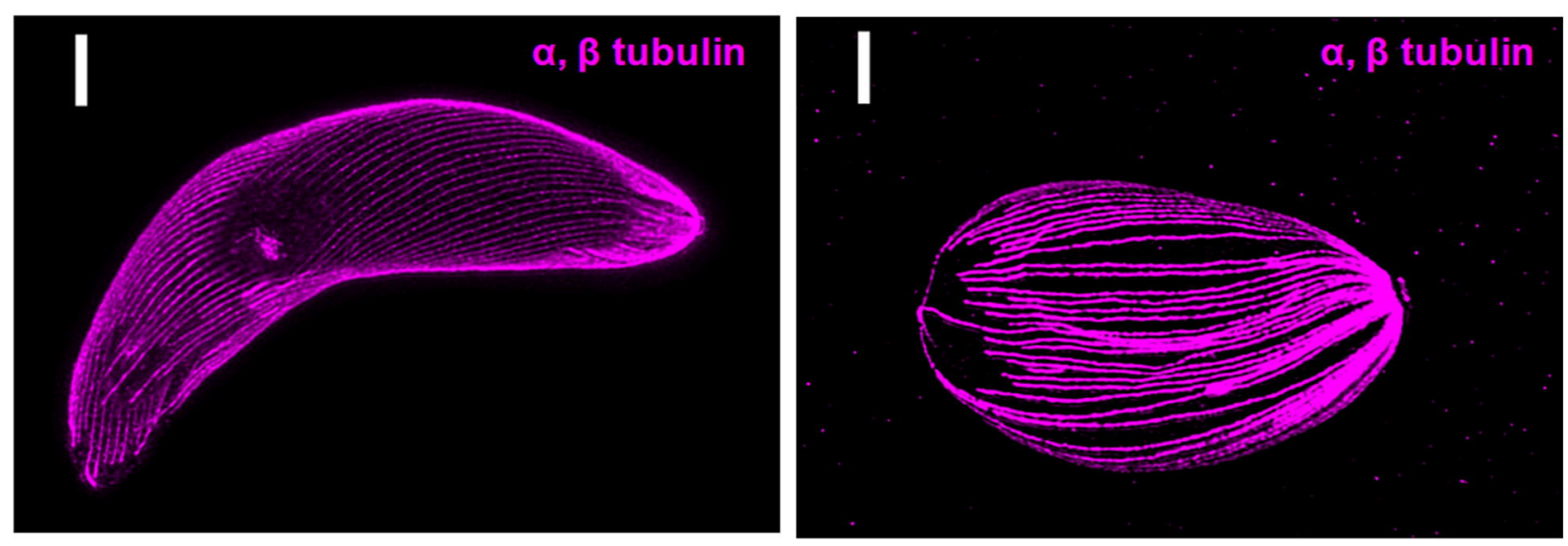

B

WTGFP
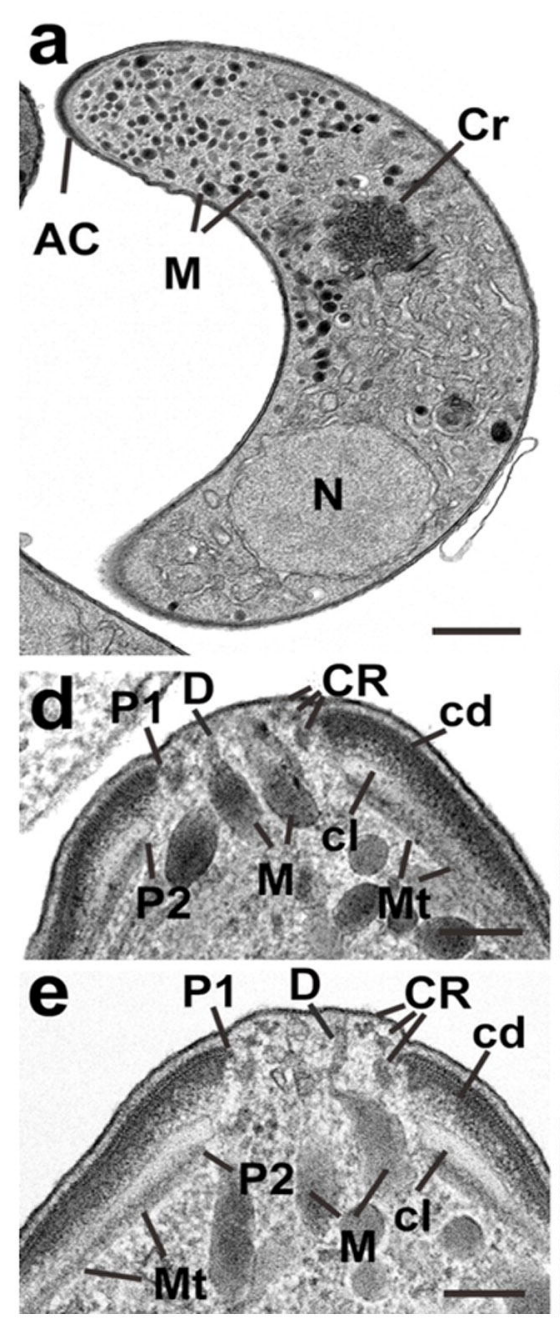

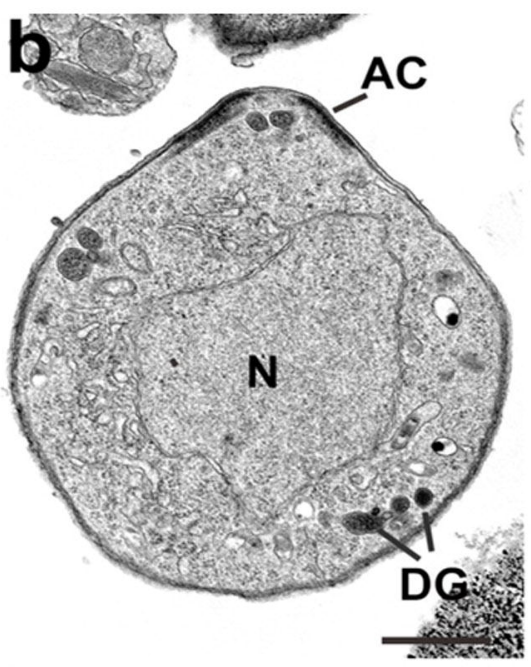

f PM cd IMC h PM
$\Delta$ kinesin-20
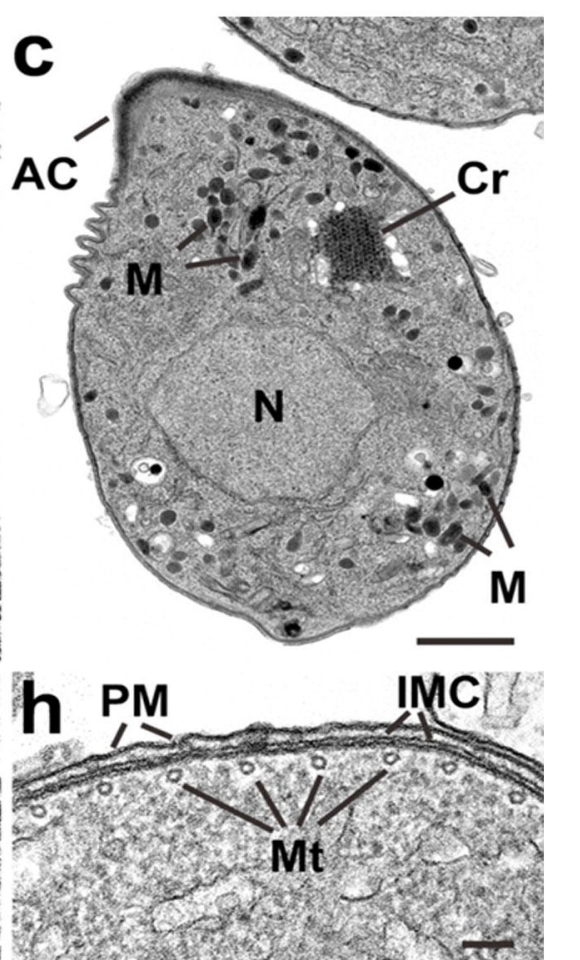
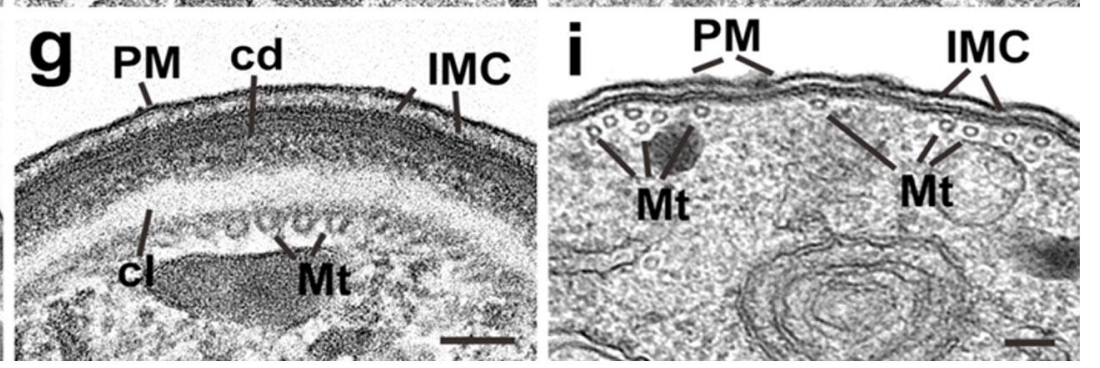
Asexual stages-Mitosis

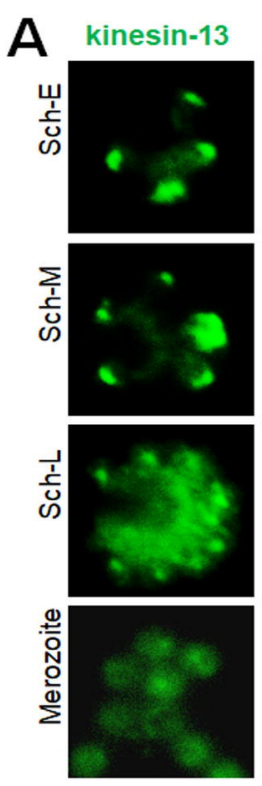

Schizogony

\section{NDC80}

Merge/Hoechst

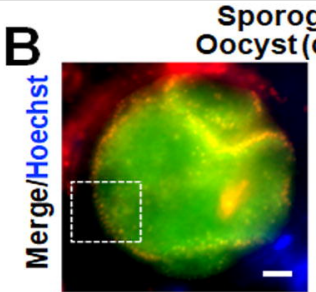

Sporogony
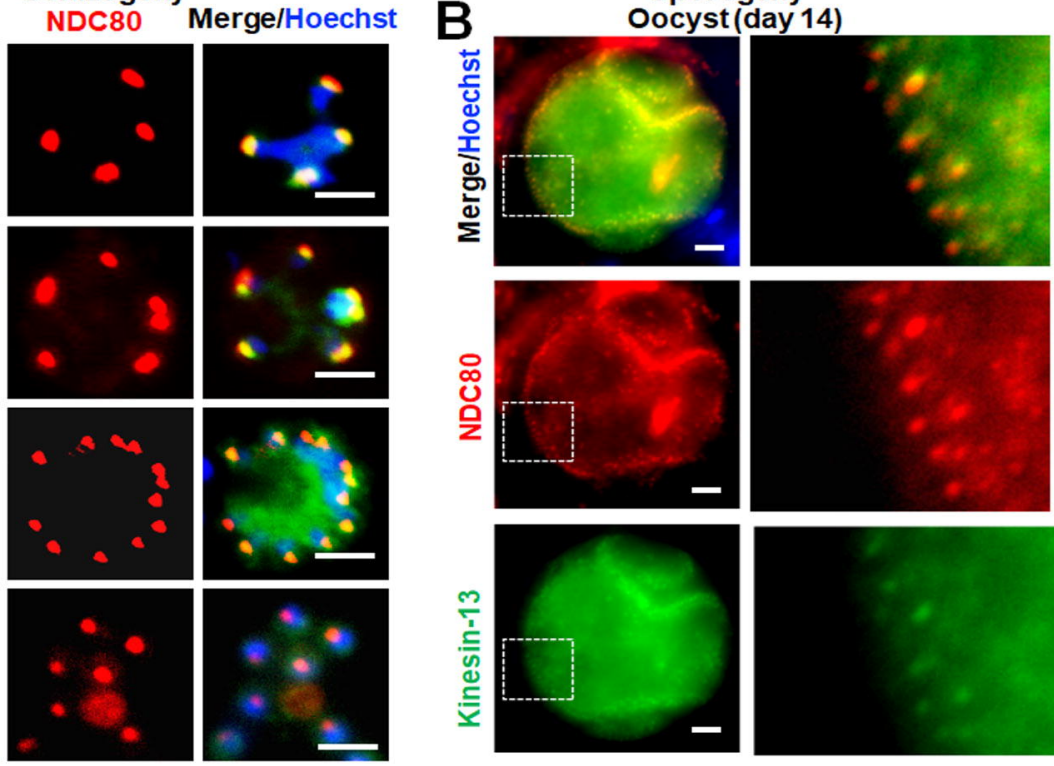

Sexual stages

Male gametogenesis-Mitosis

C kinesin-13
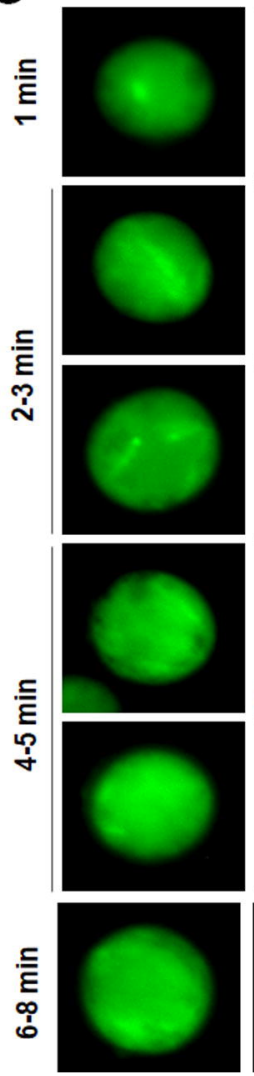

NDC80
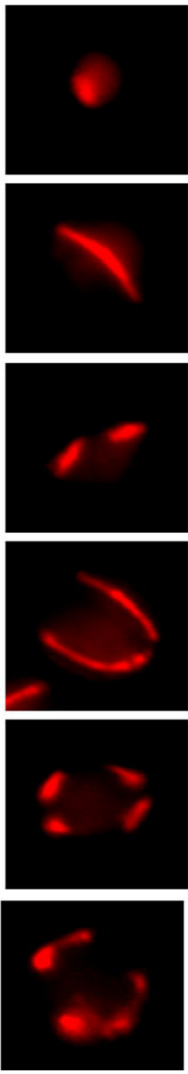
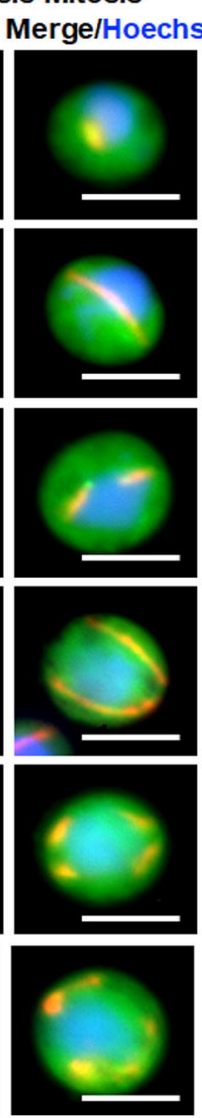

Ookinete development-Meiosis

kinesin-13 NDC80 Merge/Hoechst
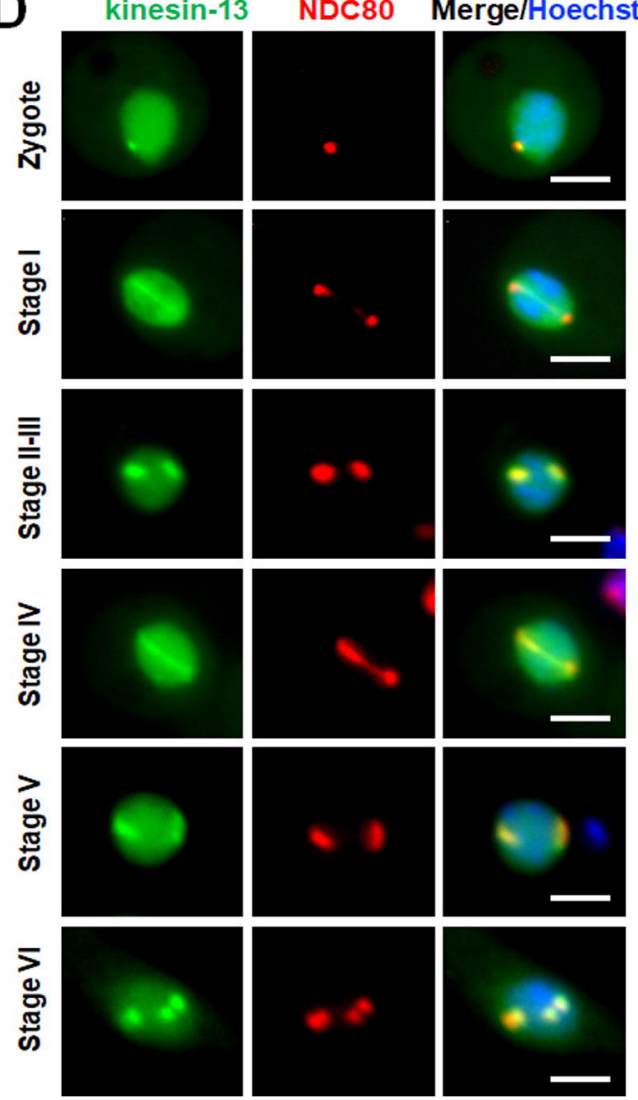
A

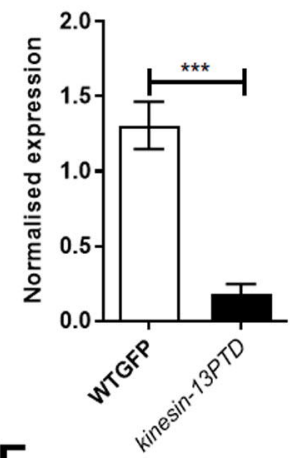

$\mathbf{F}$

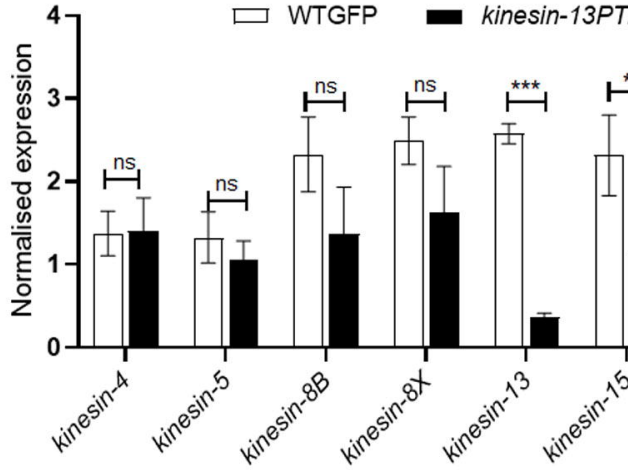

$\mathrm{H}$
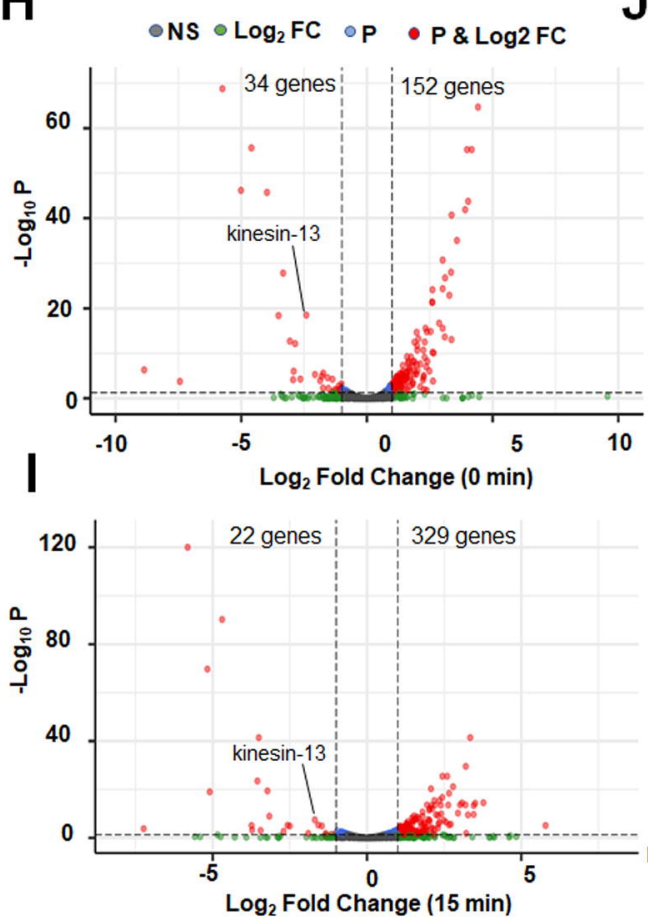

D
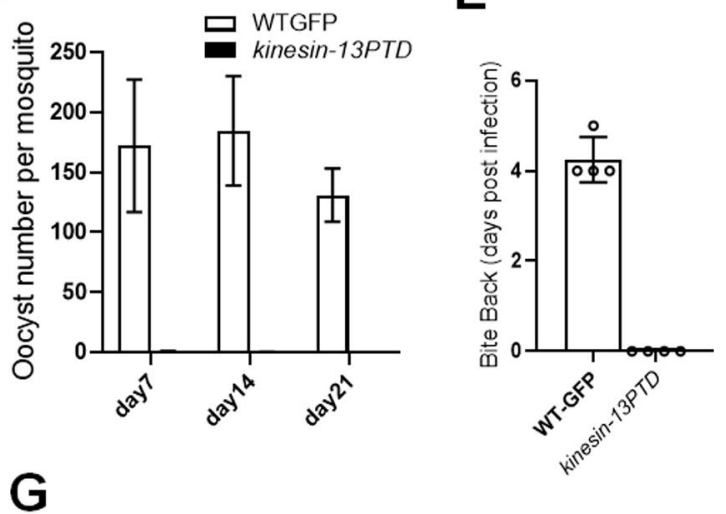

PBANKA_1458300

(kinesin-13)

kinesin-13PTD

(0 min)

kinesin-13PTD

(15 $\mathrm{min})$

WT-GFP

(0 $\mathrm{min})$

\section{WT-GFP}

(15 $\mathrm{min})$
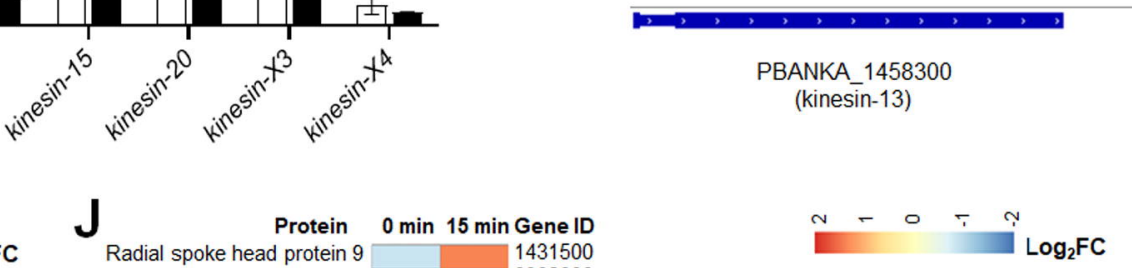

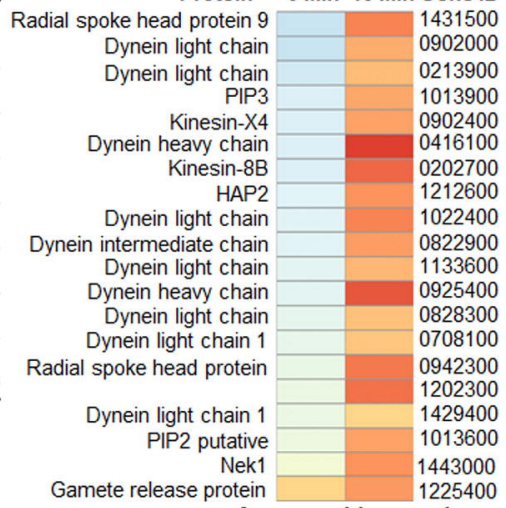

Axoneme biogenesis

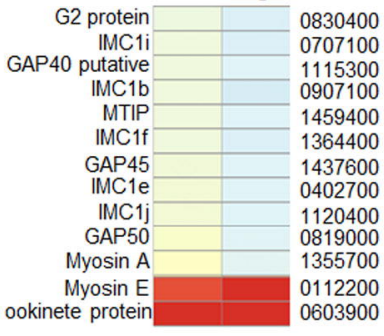

IMC/glideosome
Protein 0 min 15 min Gene ID MORN repeat protein putative $\quad 0302900$ MORN repeat protein putative MORN repeat protein putative MAP2

RAD5 putative Condensin-2-H2 putative

SPC25 putative

Protein kinase putative Condensin-2 -G2 putative 1208800 1018200 0933700 1356400 1312000 0412100 0835600 1358800 0407200 1414500 0301800 1304000 1344300 1230200 0615200 0414300 NUF2 putative PCNA1 putative MCM7 putative 1137900 0803100 ORC subunit 2 putative Protein kinase putative 0803000 0921400 Protein kinase putative 

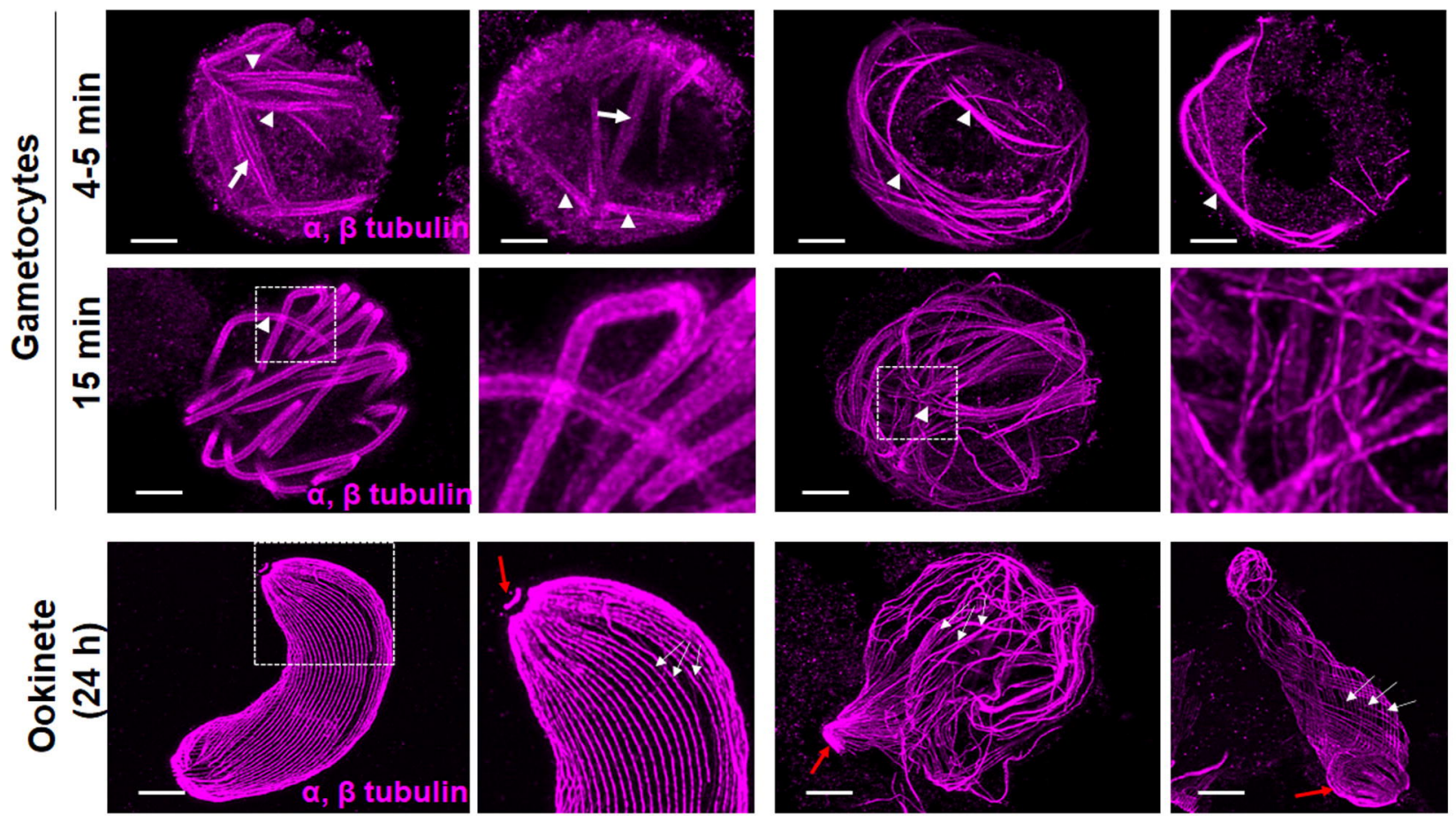

B

WTGFP

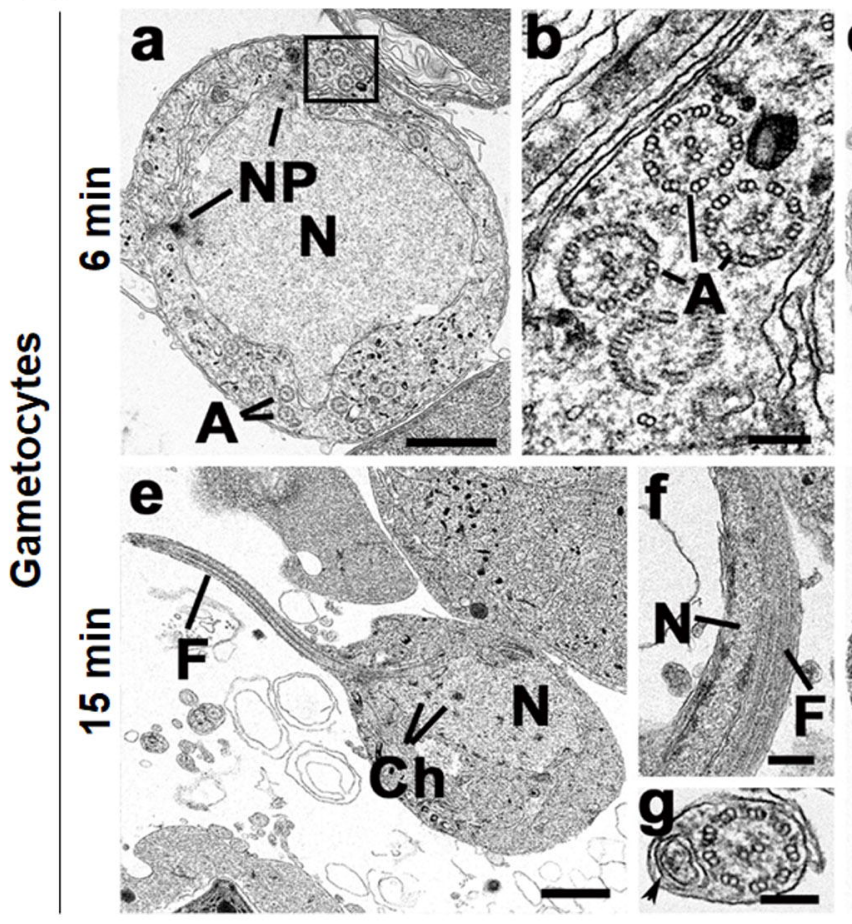

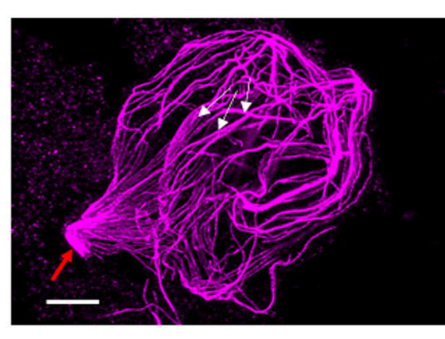

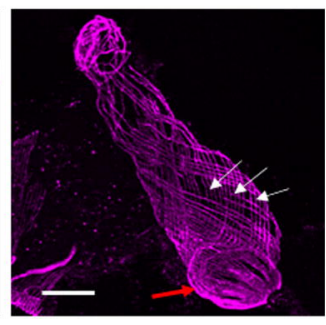

kinesin-13PTD

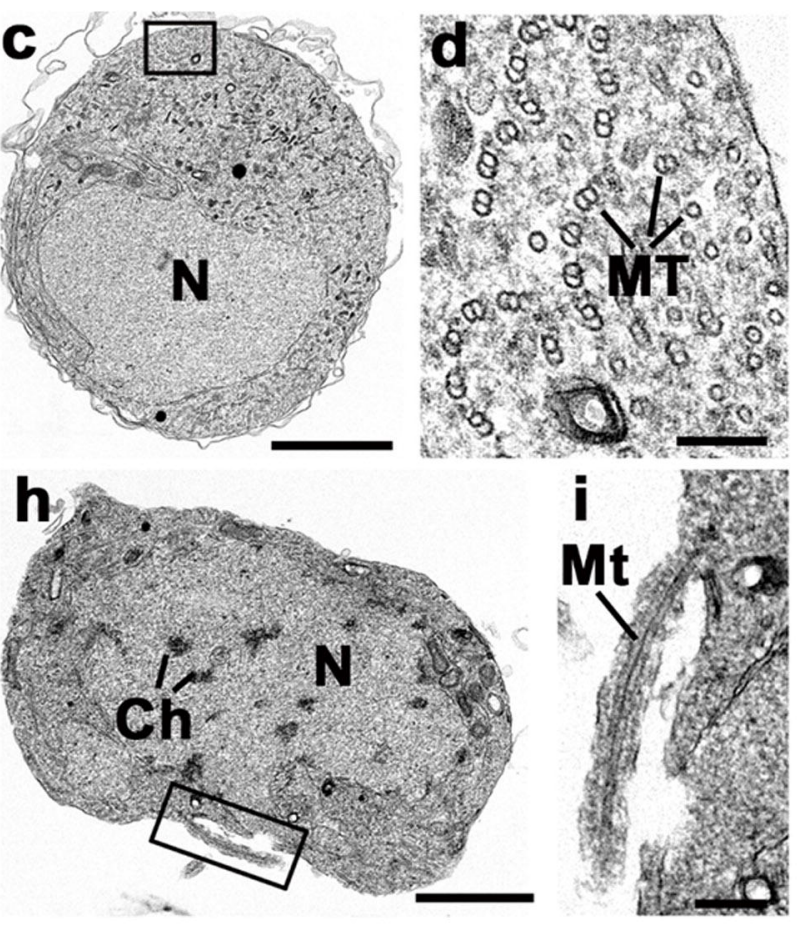




\section{Functional Analysis of kinesins in Plasmodium}

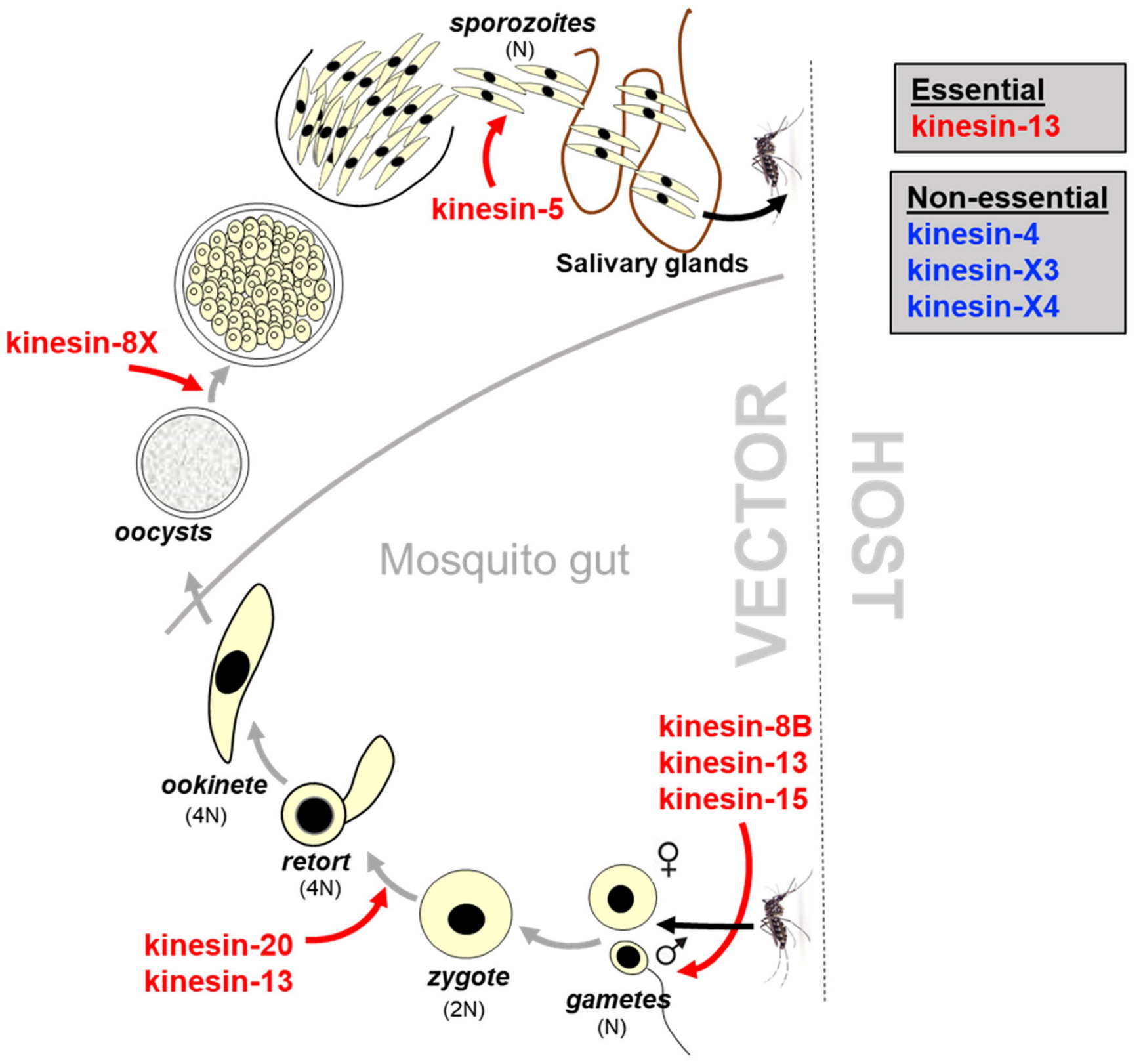

